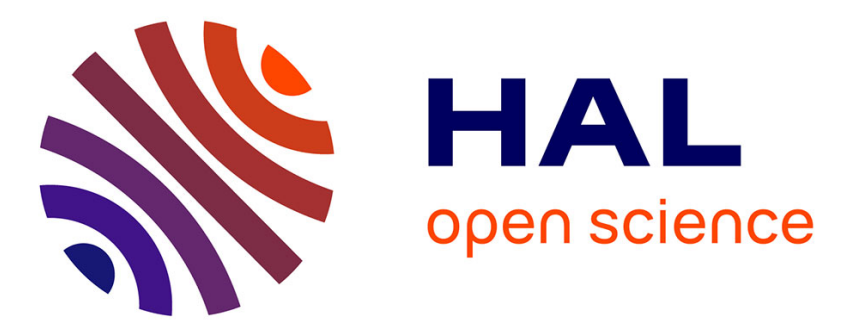

\title{
Waveform contenders for 5G: Description, analysis and comparison
}

Jean-Baptiste Doré, Robin Gerzaguet, Nicolas Cassiau, Dimitri Kténas

\section{To cite this version:}

Jean-Baptiste Doré, Robin Gerzaguet, Nicolas Cassiau, Dimitri Kténas. Waveform contenders for 5G: Description, analysis and comparison. Physical Communication, 2017, 24, pp.46 - 61. 10.1016/j.phycom.2017.05.004 . cea-01848639

\section{HAL Id: cea-01848639 https://hal-cea.archives-ouvertes.fr/cea-01848639}

Submitted on 25 Jul 2018

HAL is a multi-disciplinary open access archive for the deposit and dissemination of scientific research documents, whether they are published or not. The documents may come from teaching and research institutions in France or abroad, or from public or private research centers.
L'archive ouverte pluridisciplinaire HAL, est destinée au dépôt et à la diffusion de documents scientifiques de niveau recherche, publiés ou non, émanant des établissements d'enseignement et de recherche français ou étrangers, des laboratoires publics ou privés. 
Full length article

\title{
Waveform contenders for 5G: Description, analysis and comparison
}

\author{
Jean-Baptiste Doré *, Robin Gerzaguet, Nicolas Cassiau, Dimitri Ktenas \\ CEA-Leti Minatec, 17 avenue des Martyrs, 38054 Grenoble Cedex 9, France
}

\section{A R T I C L E I N F O}

\section{Article history:}

Received 29 March 2016

Received in revised form 15 May 2017

Accepted 17 May 2017

Available online 30 May 2017

\section{Keywords:}

Multicarrier modulation

FBMC-OQAM

FBMC-QAM

OFDM

SC-FDMA

UFMC

$5 \mathrm{G}$ cellular networks

\begin{abstract}
A B S T R A C T
$5 \mathrm{G}$ will have to cope with a high degree of heterogeneity in terms of services and requirements. Among these latter, flexible and efficient use of all available non-contiguous spectra for different network deployment scenarios is one challenge for the future 5G. To maximize spectrum efficiency, a flexible 5G air interface technology capable of mapping various services to the best suitable combinations of frequency and radio resources will also be required. In this work, a fair comparison of several $5 \mathrm{G}$ waveform candidates (UFMC, FBMC-OQAM, and FBMC-QAM) is proposed under a common framework. Spectral efficiency, power spectral density, peak to average power ratio and performance in terms of bit error rate under various realistic channel conditions are assessed. The waveforms are then compared in an asynchronous multi-user uplink transmission. Based on these results, in order to increase the spectral efficiency, a bit loading algorithm is proposed to cope with the non-uniform distribution of the interference across the carriers. The benefits of these new waveforms for the foreseen $5 \mathrm{G}$ use cases are clearly highlighted. It is also stressed that some concepts still need to be improved to achieve the full range of expected benefits of 5G.
\end{abstract}

(c) 2017 Elsevier B.V. All rights reserved.

\section{Introduction}

The fourth generation of wireless network (4G) is currently massively rolled-out but it is also known that it will quickly reach its limits. To face this issue, 3 GPP started to discuss $5 \mathrm{G}$ requirements during the RAN 5G workshop held in September 2015 leading to an emerging consensus that there will be a new, nonbackward compatible, radio access technology as part of 5G [1].

As the availability of large amount of contiguous spectrum is getting more and more difficult to guarantee, the aggregation of non-contiguous frequency bands is considered in order to meet higher data rates and/or to improve access flexibility [2]. This requirement of spectrum agility has encouraged the study of alternative multicarrier waveforms that may provide better adjacent channel leakage performance without compromising Spectral Efficiency (SE). Sporadic access has been furthermore identified as one of the significant challenges future mobile access networks will have to face. In the aim of reducing battery power consumption, a mobile node may be configured to enter a dormant state as rapidly as possible after a data transaction. This feature, called fast dormancy, has been identified as the root cause of significant signaling overhead in the cellular network [3]. Consequently, relaxed synchronization schemes have been considered to limit the

\footnotetext{
* Corresponding author.

E-mail addresses: jean-baptiste.dore@cea.fr (J.-B. Doré), robin.gerzaguet@cea.fr (R. Gerzaguet), nicolas.cassiau@cea.fr (N. Cassiau), dimitri.ktenas@cea.fr
} (D. Ktenas). required amount of signaling. This is the case for instance when the mobile node carries only a coarse knowledge of time synchronization. In the expected $5 \mathrm{G}$, the massive number of devices and the support of multi-point transmissions will imply the use of relaxed synchronization, potentially leading to strong inter-user interference.

Even though Orthogonal Frequency Division Multiplexing (OFDM) is the most prominent multi-carrier modulation technique in wireless standards for below $6 \mathrm{GHz}$ transmission, it also exhibits some intrinsic drawbacks. Firstly, an important frequency leakage is caused by its rectangular pulse shape. Secondly, the Cyclic Prefix (CP) insertion causes a SE loss. Finally, fine time and frequency synchronization is required to preserve the subcarrier orthogonality that guarantees a low level of intra and inter-cell interferences. To overcome these limitations, several alternative candidates have been intensively studied in the literature in the past few years.

In this paper, we focus on multicarrier modulation that are theoretically well localized in frequency domain. In addition to OFDM and Single Carrier Frequency Division Multiple Access (SC-FDMA), which serve as a comparison reference, we consider Universal Filtered Multi Carrier (UFMC) [4], Filter Bank Multicarrier (FBMC) Offset Quadrature Amplitude Modulation (OQAM) [5] and FBMCQAM [6]. FBMC waveforms are subcarrier-wise filtering waveforms, as each subcarrier is filtered by a time-frequency translated prototype filter. On the contrary, UFMC applies the filtering operation on a Resource Block (RB) (i.e. a group of sub-carriers) in order to decrease the filter tails. Multiple references in the 
literature focus on different receiver architectures and scenarios for these waveform candidates. In [7], OFDM and FBMC (both QAM and OQAM) are compared in terms of achievable SE in different scenarios, channel models and bandwidths. It however does not include the potential benefits of a multi-user access scheme, and the impact of FBMC parametrization. A very detailed analysis on the prototype filter choice for FBMC is done in [8]. Several filters are compared based on different design criteria but direct use-cases for waveform evaluation and comparison with classic CP-OFDM are not considered. A direct comparison between OFDM and FBMC has been done in [9]. Different prototype filters are studied, and the analysis of the time frequency behavior of the waveforms is done with the use of the ambiguity surface. In our paper, we focus on the PHYDYAS filter as it has been designed in frequency domain, and it offers very low Out of Band (OOB) leakage. This deep analysis provides a great insight on the structure of the interference but again, this paper does not provide evaluation results in typical use-case scenarios. A Bit Error Rate analysis of OFDM, FBMC and UFMC is performed in [10] with a typical ETU channel model. However, other important Key Performance Indicators (KPI) (related to frequency containment and power management) are not assessed. Some studies have also been focused on millimeter wave applications which is of importance in foreseen $5 \mathrm{G}$ scenarios [11]. In [12], millimeter RF impairments impact is studied for both OFDM and Discrete Fourier Transform (DFT)-based waveform. It shows the potential benefits of DFT precoding, but it however does not consider other popular (and filtered) waveforms such as FBMC and UFMC. Finally an introduction of promising single and multi-carrier waveforms has been done in [13]. Detailed evaluation results are nevertheless not included.

The main purpose of this paper is to propose a common framework for the evaluation of the different waveform candidates in different representative scenarios. After a brief introduction to the waveforms, the evaluation is done in a three step analysis. First, we compare the waveforms regarding different representative criteria. We then assess the waveforms for different frequency selective channel models, together with different parametrizations. We finally study the multi-user scenario performance. The main contributions of the paper, in addition to be a comprehensive study of some of the most promising multicarrier waveforms in expected $5 G$ scenarios, are:

- to propose a unified scenario to evaluate and compare the different waveforms performance,

- to provide a deep analysis of the Power Spectral Density (PSD), the Peak to Average Power Ratio (PAPR), and the performance in a multi-user access scenario. (From this, we provide an optimization analysis that improves the SE. We show the advantages of a well localized waveform in frequency domain.),

- to derive a performance comparison between the waveforms in typical channel models (we also compare and analyze the performance of two different FBMC receiver architectures),

- finally, to study the impact and the benefits of reducing the Fast Fourier Transform (FFT) size for FBMC, especially when the Frequency Spreading (FS) architecture is used as it improves the spectral resolution of the equalizer.

In this work, the multi-user interference is modeled by a bandwidth shared between three users. The user of interest is assumed to be perfectly synchronized (in both time and frequency domains) with its serving base station (BS). The two other users are not synchronized with the serving BS and thus interfere with the user of interest. The performance of the candidate waveforms (UFMC, FBMC-OQAM and FBMC-QAM) are compared and discussed for several delays and carrier frequency offsets, for different number of guard carriers, and according to different waveform parameters such as the size of the FFT. The baselines for comparison are the Long Term Evolution (LTE) waveforms, namely CP-OFDM and SCFDMA. In order to investigate the distribution of the interference inside a physical RB, the studied metric is the Mean Square Error (MSE) per carrier at the receiver side, after demodulation. Next, we explore how the SE could be increased by properly choosing, for each carrier, the modulation order.

We also compare in this paper the SE, the PSD and the PAPR for each waveform candidate. Furthermore Bit Error Rate (BER) performance are assessed for representative 3GPP channel models for the purpose of getting insights on the robustness of receivers to realistic channel conditions. This paper thus proposes a fair comparison regarding different criteria that are representative for benchmarking $5 \mathrm{G}$ waveforms.

The remainder of the paper is organized as follows. The main 5G waveform candidates along with their associated transceivers are described in Section 2. A detailed comparison of the waveforms is then performed in Section 3 with respect to the following KPI: PSD, PAPR, SE and BER. In Section 4, the waveform performances in terms of MSE are compared in the case of a typical multiuser asynchronous access scenario. An optimization of the SE, based on bit loading algorithm, is proposed and its performance are discussed. Eventually the last section draws some conclusions.

\section{Waveforms description}

In this section, we briefly introduce the main waveform candidates for $5 G$.

\subsection{Notations}

Bold letters denote vectors and matrices. Upper-case and lowercase letters denote frequency domain and time domain variables respectively. The following notations were used:

- $(.)^{H}$ Hermitian transform

- $E[$. ] Expectation operator

$\mathbf{F}_{\mathbf{N}}$ stands for the DFT matrix defined as:

$\mathbf{F}_{\mathbf{N}}=\frac{1}{\sqrt{N}}\left[\begin{array}{ccccc}1 & 1 & 1 & \ldots & 1 \\ 1 & w_{N} & w_{N}^{2} & \ldots & w_{N}^{N-1} \\ \vdots & \vdots & \vdots & & \vdots \\ 1 & w_{N}^{N-1} & w_{N}^{2(N-1)} & \ldots & w_{N}^{(N-1)(N-1)}\end{array}\right]$

where $w_{N}=e^{j \frac{2 \pi}{N}}$

\section{2. $C P-O F D M$}

In classic CP-OFDM, the carriers are modulated at a frequency $k / N$, using rectangular shapes in the time domain. The mapping/demapping process can be done with an Inverse FFT (IFFT)/FFT and thus allows an architecture with low complexity (see Fig. 1). The insertion of the $\mathrm{CP}$ guarantees circularity of the OFDM symbol. A one tap per carrier equalization process can be applied when a good dimensioning of the $\mathrm{CP}$ with respect to the channel delay spread is done. The simplicity of the OFDM waveform, which led to its selection for numerous wireless standards, is however counterbalanced by its important Adjacent Channel Leakage Ratio (ACLR), due to the use of sinc shapes in the frequency domain, and an important PAPR due to its multicarrier nature. It also requires a good synchronization in time domain in the context of multiple access scenario to preserve the orthogonality of the carriers.

An OFDM symbol can be expressed as:

$\mathbf{x}_{\mathbf{k}}=\mathbf{r}_{\mathbf{N}+\mathbf{N}_{\mathbf{c}}, \mathbf{N}} \mathbf{F}_{\mathbf{N}}{ }^{H} \mathbf{X}_{\mathbf{k}}$ 


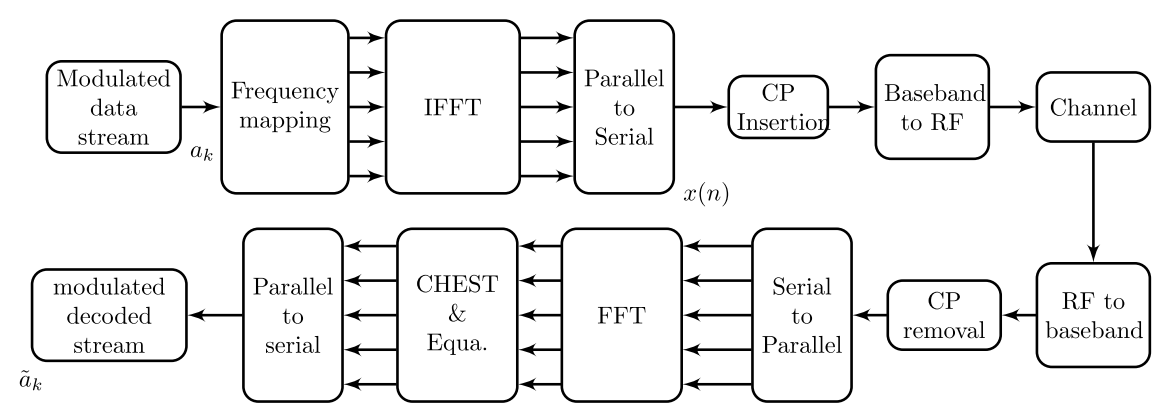

Fig. 1. OFDM transceiver block diagram. CHEST stands for CHannel ESTimation.

with $\mathbf{X}_{\mathbf{k}}$ being the $k$ th symbol to transmit, $N_{c}$ the length of the cyclic prefix and $\mathbf{r}_{\mathbf{N}+\mathbf{N}_{\mathbf{c}}, \mathbf{N}}$ the $\mathrm{CP}$ insertion matrix, defined as

$\mathbf{r}_{\mathrm{N}+\mathbf{N}_{\mathbf{c}}, \mathbf{N}}=\left[\begin{array}{lll}\mathbf{0}_{\mathrm{N}_{\mathbf{c}}, \mathrm{N}-\mathbf{N}_{\mathbf{c}}} & & \mathbf{1}_{\mathbf{N}_{\mathbf{c}}} \\ & \mathbf{1}_{\mathrm{N}} & \end{array}\right]$

where $\mathbf{0}_{\mathbf{N}_{\mathbf{c}}, \mathbf{N}-\mathbf{N}_{\mathbf{c}}}$ is the zero-matrix of size $N_{c} \times\left(N-N_{c}\right)$ and $\mathbf{1}_{\mathbf{N}}$ is the identity matrix of size $N \times N$.

\subsection{SC-FDMA}

CP-OFDM exhibits a high PAPR, which is problematic in terms of transmit power efficiency and constraints for the power amplifier. In the uplink (UL) mode of 3GPP-LTE, this issue has been solved by the use of SC-FDMA which is based on classic CP-OFDM with a precoding stage [14] (see Fig. 2). The precoding stage is a DFT applied before the IFFT stage at the transmitter. In the receiver stage, an IDFT is applied after the FFT to recover the data.

A SC-FDMA symbol can be expressed as

$\mathbf{x}_{\mathbf{k}}=\mathbf{r}_{\mathbf{N}+\mathbf{N}_{\mathbf{c}}, \mathbf{N}} \mathbf{F}_{\mathbf{N}}{ }^{H} \mathbf{D} \mathbf{X}_{\mathbf{k}}$

where $\mathbf{D}$ is the blockwise spreading matrix that contains DFT coefficients.

\subsection{FBMC-OQAM}

With FBMC, a set of parallel data symbols are transmitted through a bank of modulated filters. The choice of the prototype filter controls the localization in frequency of the generated pulse and can provide better adjacent channel leakage performance in comparison to OFDM. OQAM combined with Nyquist constraints on the prototype filter is used to guarantee orthogonality (in the real field) between adjacent symbols and adjacent carriers while providing maximum SE [15]. The duration $L$ of the prototype filter is a multiple of the size of the FFT, $N$, so that $L=K N$. $K$ is an integer and usually referred as to the overlapping factor. Frequency sampling technique is often considered to design the prototype filter. The technique has been proven simple and yet very efficient to build an almost optimal filter as a function of $K$. The FBMC transmitter-receiver structure can be efficiently implemented using IFFTs or FFTs combined with a PolyPhase Network (PPN) [15]. Frequency Spreading approach (FS-FBMC) has been recently proposed in [16] and [17] as an alternative to PPN-FBMC. This technique is inspired by the frequency sampling technique used to design the prototype filter. With this approach, the number of nonzero samples in the frequency response is given by $P=2 K-1$. For $K=2$, the frequency domain pulse response coefficients are equal to [15]:

$G_{0}=1 ; G_{1}=G_{-1}=\frac{\sqrt{2}}{2}$.
For $K=3$ :

$G_{0}=1 ; G_{1}=G_{-1}=0.911438 ;$

$G_{2}=G_{-2}=\sqrt{1-G_{1}^{2}}$

and for $K=4$ :

$G_{0}=1 ; G_{1}=G_{-1}=0.971960$;

$G_{2}=G_{-2}=\frac{\sqrt{2}}{2} ; G_{3}=G_{-3}=\sqrt{1-G_{1}^{2}}$.

The impulse response of the prototype filter is then given by:

$h(t)=G_{0}+2 \sum_{k=1}^{K-1}(-1)^{k} G_{k} \cos \left(\frac{2 \pi k}{K N}(t+1)\right)$.

With frequency spreading technique, symbols are spread over $P$ carriers by filtering the output of the OQAM process by the frequency domain pulse response. The output is then processed through an IFFT of size $K N$. OQAM precoding imposes that real and pure imaginary symbol values alternate on successive carrier frequencies and on successive transmitted symbols for any given carrier. This precoding guarantees orthogonality between adjacent carriers since the coefficients of the prototype filter are real. The output of the IFFT is converted through a Parallel-to-Serial $(\mathrm{P} / \mathrm{S})$ conversion and is accumulated with the following IFFT output data block stream delayed by $N / 2$. This $\mathrm{P} / \mathrm{S}$ conversion is called overlapand-sum. Once the transient period is over, $2 K$ of the $K N$-IFFT output samples are added together at any given time. Therefore the time domain signal can be expressed as [18]:

$\mathbf{y}_{\mathbf{k}}=\mathbf{F}_{\mathbf{N}}{ }^{H} \mathbf{G} \mathbf{X}_{\mathbf{k}}+\sum_{p=1}^{2 K-1} \mathbf{Q}_{-\frac{\mathbf{p N}}{2}} \mathbf{F}_{\mathbf{N}}{ }^{H} \mathbf{G} \mathbf{X}_{\mathbf{k}-\mathbf{p}}+\mathbf{Q}_{\frac{\mathbf{p N}}{\mathbf{2}}} \mathbf{F}_{\mathbf{N}}{ }^{H} \mathbf{G} \mathbf{X}_{\mathbf{k}+\mathbf{p}}$

where matrix $\mathbf{G}$ is the prototype filter matrix. $\mathbf{Q}_{\mathbf{x}}$ introduces $x$ samples delay between the block of samples. Eq. (6) represents the sum of the $2 \times(2 K-1)+1$ filtered FBMC-OQAM symbols that overlap over time. The corresponding transceiver is depicted Fig. 3. By using the property of the prototype filter and the property of the spreading process (in frequency domain), it is possible to derive a closed form expression of the time domain signal illustrating the use of a Fourier transform of size $N$ combined with a PPN filter. This formulation is interesting for practical implementation as demonstrated in [15].

At the receiver side, the dual operation of the overlap-and-sum operation of the transmitter is a sliding window in the time domain that selects $K N$ points every $N / 2$ samples. A FFT is then applied on every block of $K N$ selected points. Equalization is applied using a single tap equalizer and is followed by the filtering with the prototype matched filter (Fig. 4(a)). Data at the output of the matched filter is then demapped to compute a log-likelihood ratio for the input of the inner decoder. Because the size of the FFT is $K$ times larger than the multicarrier symbol time period, the signal at the output of the FFT is oversampled by a factor $K$ compared to the 


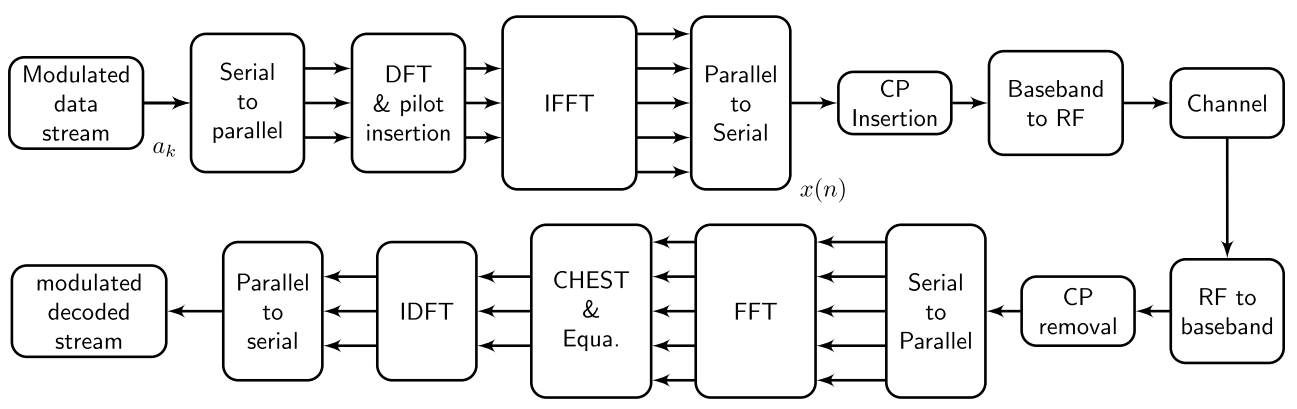

Fig. 2. SC-FDMA transceiver block diagram.

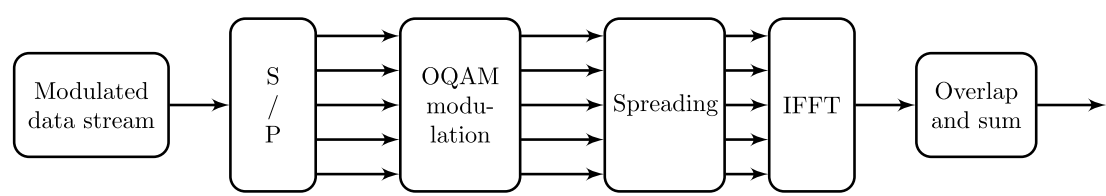

Fig. 3. FBMC-OQAM transmitter block diagram.

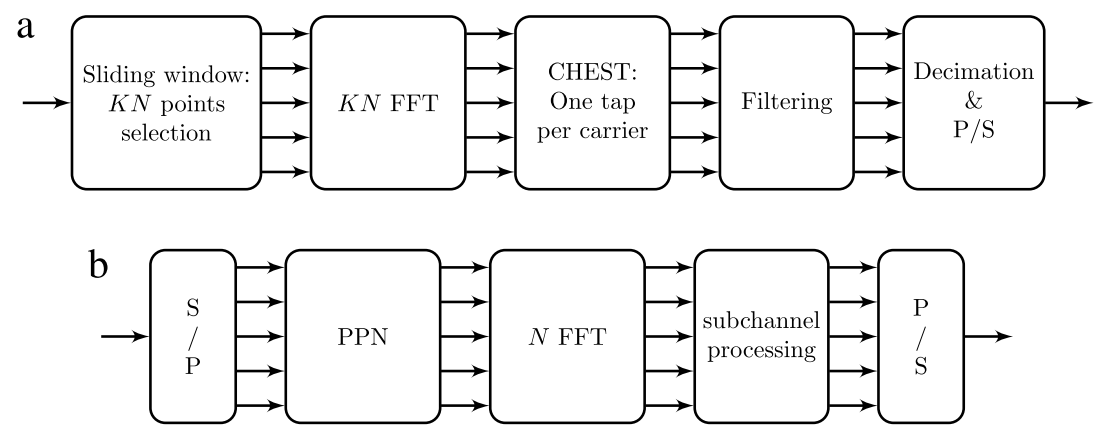

Fig. 4. FBMC-OQAM receiver block diagram. (a) using FS approach (b) using classical PPN approach.

carrier spacing. This property gives FS-FBMC receiver a significant advantage for channels with large delay spread. It also suggests that the intercarrier spacing could be reduced; this property will be discussed in the following. Of course the classical PPN receiver scheme can also be applied [15] (Fig. 4(b)).

\subsection{FBMC-QAM}

The filtering of adjacent carrier by the prototype filter overlaps in the classical FBMC-OQAM system and consequently an intrinsic interference occurs (addressed by the use of OQAM modulation instead of QAM). The main idea behind FBMC-QAM is to design two sets of orthogonal prototype filters. These filters are optimized to minimize or null the intrinsic interference between the evennumbered and the odd-numbered carriers [6]. In order to satisfy the orthogonality conditions, the FBMC-QAM scheme proposed in [6] performs a block-wise permutation of the time domain signal for the odd-numbered sub-carrier symbols after the overlap and sum processing. The corresponding transceiver is depicted in Fig. 5. It should be mentioned that due to the block-wise permutation, the waveform is no longer well localized in frequency. It is possible to mitigate this effect by applying a "smoothing" filter [19]. In that case the frequency localization of the waveform is managed but the orthogonality condition is broken. As a consequence, a tradeoff should be found between a good frequency localization and the level of interference generated.
A FBMC-QAM symbol $\mathbf{y}_{\mathbf{k}}$ can be expressed as follows:

$$
\begin{aligned}
\mathbf{y}_{\mathbf{k}}= & \mathbf{F}_{\mathbf{N}}{ }^{H} \mathbf{G} \mathbf{X}_{\mathbf{k}}^{\mathbf{0}}+\sum_{p=1}^{K-1} \mathbf{Q}_{-\mathbf{p N}} \mathbf{F}_{\mathbf{N}}{ }^{H} \mathbf{G} \mathbf{X}_{\mathbf{k}-\mathbf{p}}^{\mathbf{0}}+\mathbf{Q}_{\mathbf{p N}} \mathbf{F}_{\mathbf{N}}{ }^{H} \mathbf{G} \mathbf{X}_{\mathbf{k}+\mathbf{p}}^{\mathbf{0}} \\
& +\mathbf{\Omega} \mathbf{F}_{\mathbf{N}}{ }^{H} \mathbf{G} \mathbf{X}_{\mathbf{k}}^{\mathbf{1}}+\boldsymbol{\Omega} \sum_{p=1}^{K-1} \mathbf{Q}_{-\mathbf{p N}} \mathbf{F}_{\mathbf{N}}{ }^{H} \mathbf{G} \mathbf{X}_{\mathbf{k}-\mathbf{p}}^{\mathbf{1}}+\mathbf{Q}_{\mathbf{p N}} \mathbf{F}_{\mathbf{N}}{ }^{H} \mathbf{G} \mathbf{X}_{\mathbf{k}+\mathbf{p}}^{\mathbf{1}}
\end{aligned}
$$

where matrix $\boldsymbol{\Omega}$ stands for the blockwise interleaving. $\mathbf{X}_{\mathbf{k}+\mathbf{p}}^{\mathbf{0}}$ and $\mathbf{X}_{\mathbf{k}+\mathbf{p}}^{\mathbf{1}}$ are the matrices that model the data on even and odd carriers.

The classical PPN receiver scheme is suggested by the authors of [6]. However, in this work, we propose to extend the FS-based receiver scheme to the FBMC-QAM. In that case, a KN FFT is processed every blocks of $N$ samples generating $K N$ points. The FFT is applied to two versions of the received stream (in the natural order and in an interleaved order). Outputs of the FFTs are then equalized and filtered in the frequency domain before being selected according to the carrier parity (odd and even). The corresponding receiver is depicted Fig. 6.

\subsection{UFMC}

In UFMC a group of $n_{i}$ carriers is filtered in the frequency domain. This subband filtering operation is motivated by the fact that the smallest unit used by the scheduling algorithm in frequency domain in 3GPP LTE is a RB, which is a group of 12 carriers [4]. The filtering operation thus leads to a lower ACL. Depending on 


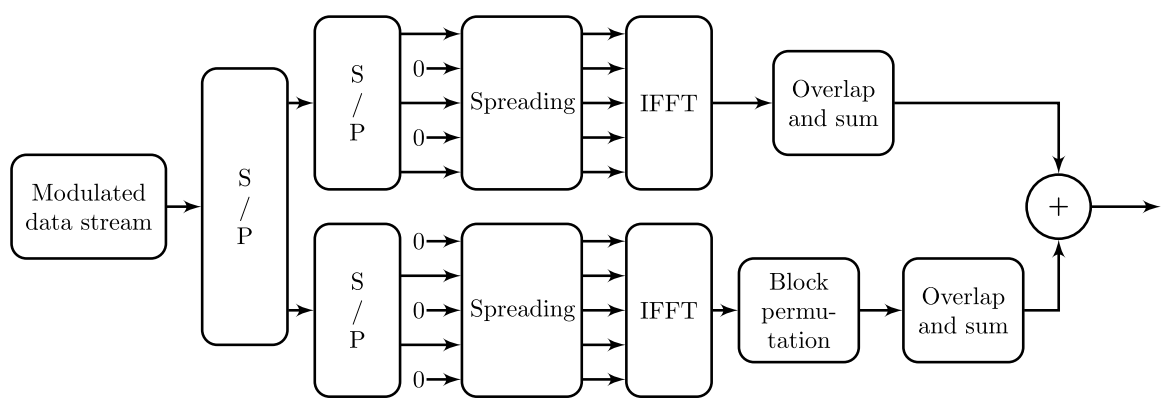

Fig. 5. FBMC-QAM transmitter block diagram.

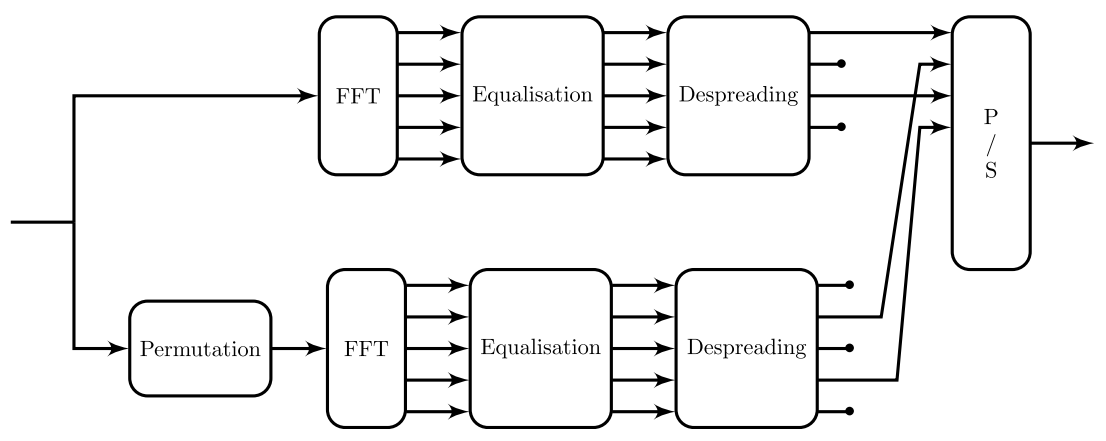

Fig. 6. FBMC-QAM receiver block diagram with FS implementation.

the filter parameters, UFMC can be changed into classic OFDM or single carrier [20]. The transceiver [21] (see Fig. 7) is composed of $B$ subband filtering operations (combined with frequency mapping), that modulate the $B$ data blocks. The obtained signals are then mixed. There is no CP insertion, but there is a SE loss due to the transient state of the shaping filter (of length $L$ ) introduced by the convolution (see Section 3.2). The time-domain transmit vector $x$ of length $1 \times N_{F F T}+L-1$ for a multicarrier symbol of user $k$ is the superimposition of its subband filtered components, and can be expressed as [22]:

$\mathbf{x}_{\mathbf{k}}=\sum_{i=1}^{B} \mathbf{C}_{\mathbf{i}, \mathbf{k}} \mathbf{F}_{\mathbf{i}, \mathbf{k}} \mathbf{S}_{\mathbf{i}, \mathbf{k}}$,

where $\mathbf{S}_{\mathbf{i}, \mathbf{k}}$ is the data vector for the block $i$, of size $1 \times n_{i}, \mathbf{F}_{\mathbf{i}, \mathbf{k}}$ is the IFFT matrix of size $N_{F F T} \times n_{i}$ with appropriate frequency mapping, and $\mathbf{C}_{\mathbf{i}, \mathbf{k}}$ is the filtering matrix of size $\left(N_{F F T}+L-1\right) \times N_{F F T}$.

Each received symbol, of length $N_{F F T}+L-1$, is padded with zeros before a $2 N_{F F T}$ point FFT is applied, which is next decimated by a factor 2 to recover the data [21]. It is also possible to insert before the FFT a windowing stage. This stage introduces interference between carriers but is interesting when asynchronous UL scheme is considered as it helps to separate contiguous users, and thus can improve the performance, as shown in Section 4. In the rest of the paper, we consider $n_{i}=12$, a Dolph-Chebyshev shaping filter with length $L=73$ (to ensure the same SE as CP-OFDM) with an attenuation factor of $40 \mathrm{~dB}$ as in [20,22].

\section{Waveform comparisons}

Several waveform candidates have been introduced in the previous section. We now compare their performance with respect to several criteria. We first compute the PSD, in order to stress the impact of FBMC parametrization in terms of OOB leakage. We then study the PAPR for the different waveforms. Finally, we assess the performance of the different $5 \mathrm{G}$ candidates in terms of BER for two typical multipath channels.

\subsection{Power spectral density}

We compare in this section the PSD of the studied waveforms. The spectral location of a waveform is intrinsically linked to the spectrum reuse and to the support of potential co-existence of different services. To better stress the impact of adjacent channel leakage, we consider a scenario with two frequency blocks of $540 \mathrm{kHz}$ (3RBs) spaced by a hole of $180 \mathrm{kHz}$ (1RB). The PSD of the resulting signal is depicted in Fig. 8. FBMC-QAM shows higher OOB leakage compared to classic CP-OFDM due to the block-wise permutation and to the discontinuous transition. The application of a smoothing filter could reduce the OOB radiation but in that case the complex orthogonality would be broken.

The best spectral location is obtained with FBMC-OQAM with a FFT size of 1024 and an overlapping factor $K=4$. UFMC, due to the block filtering, has a lower OOB leakage compared to OFDM, but is outperformed by FBMC-OQAM. In case of FBMC-OQAM, the smaller the FFT length, the better the time location; therefore, reducing the FFT size (increasing the inter carrier spacing) relaxes the frequency localization. However, even with a FFT size divided by 4 (i.e. a FFT size of 256 instead of 1024), the OOB leakage is still lower compared to the other waveform candidates.

We depict in Fig. 9 the PSD of OFDM, UFMC and FBMC-OQAM for several values of $K$. The overlapping factor directly controls the location in the frequency domain, and the spectral containment can be heavily relaxed with the decreasing of $K$. For $K=3$, the spectral location is still very good and outperforms UFMC. For $K=2$, the OOB leakage increases, and is only $10 \mathrm{~dB}$ lower than OFDM. A small overlapping factor induces a lower pulse duration, making this configuration interesting for short burst transmission or robustness against channel with small coherence time. It is also noticeable that even for $K=2$, the FBMC prototype filter shows good performance if a small frequency guard band (here one $\mathrm{RB}$ ) is inserted between two adjacent users, as the OOB for $K=2$ to 4 is always lower compared to UFMC. 


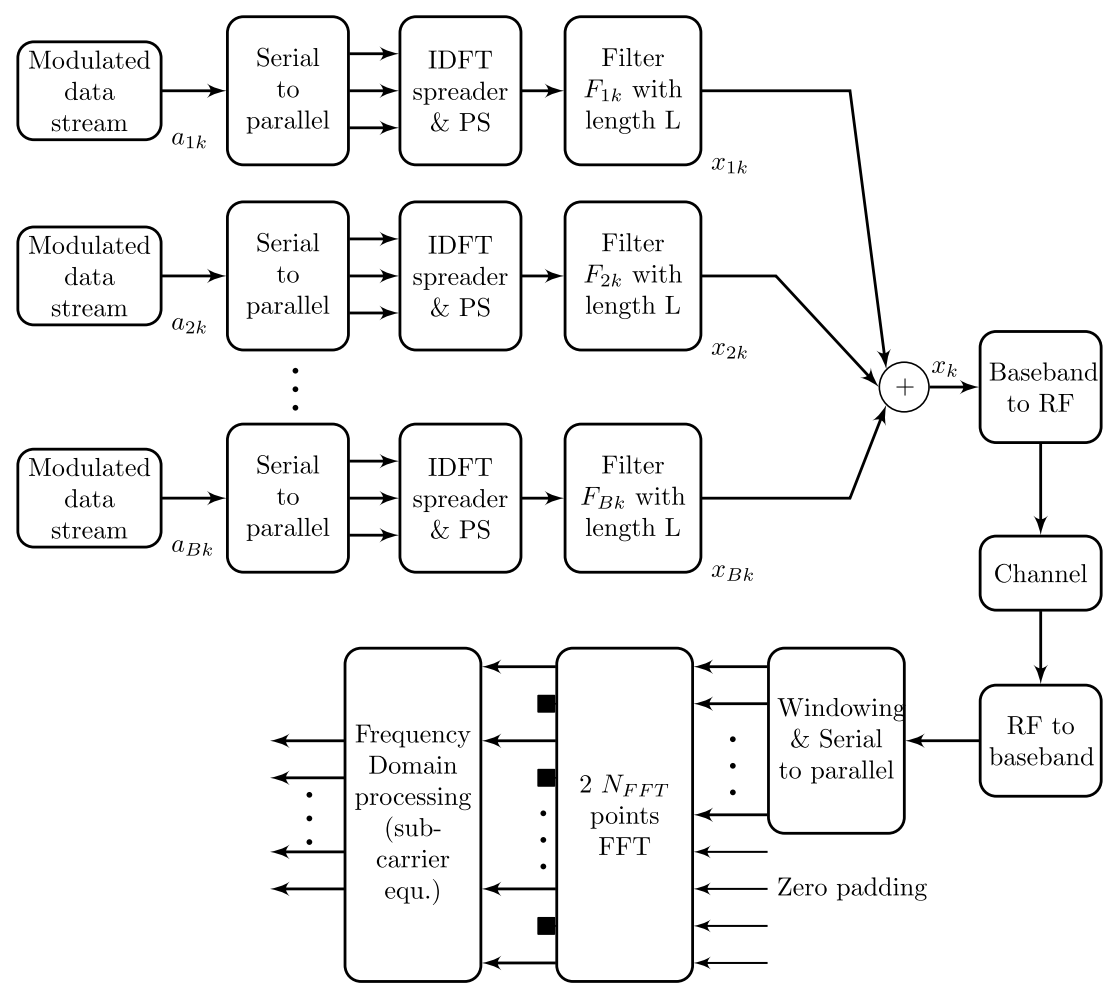

Fig. 7. UFMC transceiver block diagram.

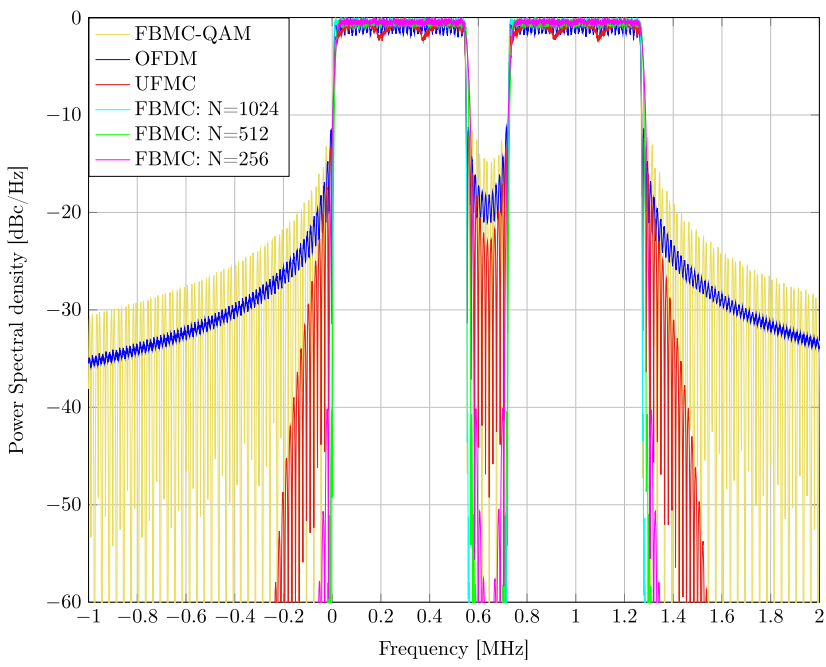

Fig. 8. Power spectral density of OFDM, UFMC, FBMC-OQAM and FBMC-QAM.

\subsection{Spectral efficiency}

First, we propose to compare the waveforms according to the SE expressed in bit per second per Hertz. In our methodology, we do not take into account the potential gain introduced by the good frequency localization of the waveforms. Indeed, when the waveforms have been designed to fulfill a very low OOB radiation constraint, few guard carriers at each side of the band allow for nearly null out-of-band radiation. An evaluation of the benefits of this approach has been demonstrated in [23].

In case of OFDM, SC-FDMA and UFMC, the SE does not depend on the burst duration and is a function of the FFT size and the modulation efficiency (which itself depends on the coding rate, the

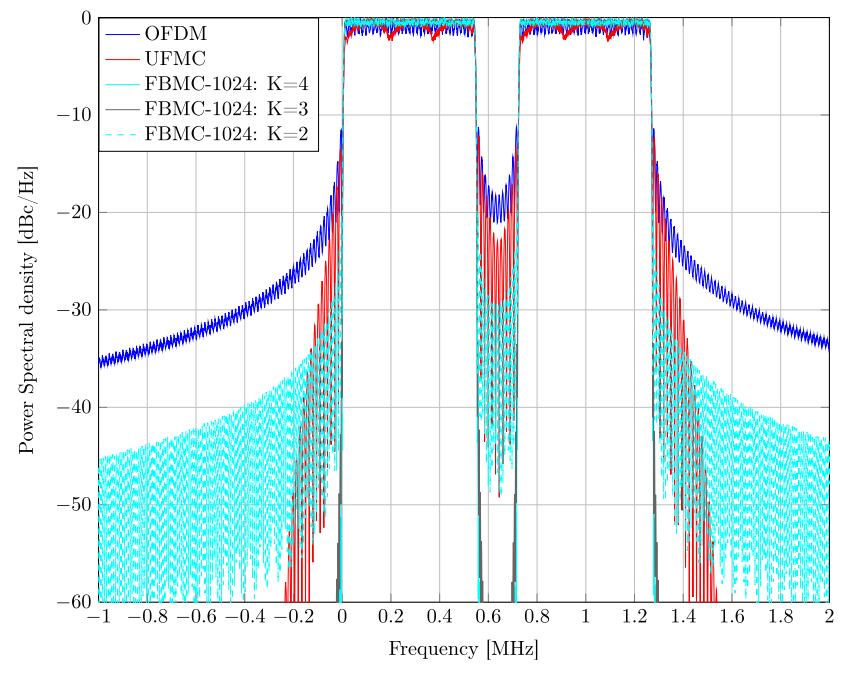

Fig. 9. Power spectral density of OFDM, UFMC and FBMC-OQAM with several values of $K$.

rate matching, the modulation order and the number of active RBs). For OFDM and SC-FDMA, the SE can be described as follows:

$\eta_{\text {OFDM }}=\eta_{S C-F D M A}=\frac{N}{N_{c}+N} \eta$

where $\eta$ is the modulation efficiency (including modulation order, coding rate and number of active carriers). In case of UFMC, the SE depends on the duration of the transient state $L$ of the shaping filter:

$\eta_{\text {UFMC }}=\frac{N}{L-1+N} \eta$.

In the literature, $L=N_{c}+1$ is a classical parametrization that ensures the same SE between UFMC and OFDM [20]. 


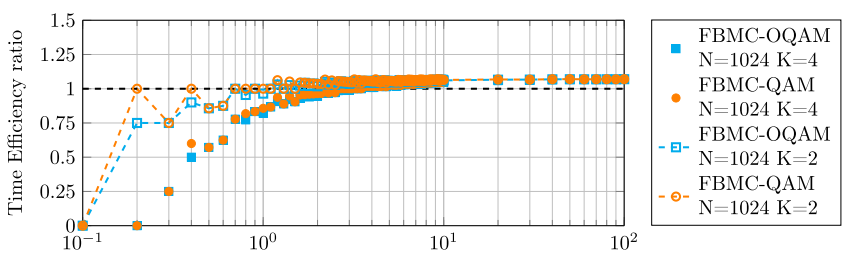

Slot duration $[\mathrm{ms}]$

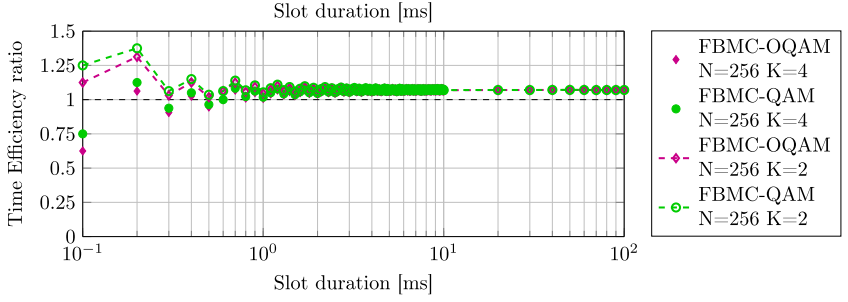

Fig. 10. Ratio efficiency between an OFDM (and UFMC) waveform with LTE $10 \mathrm{MHz}$ parameters and FBMC-OQAM/QAM waveforms for different transmission time.

As mentioned previously, the case of FBMC is more complex as the $S E$ is a function of the number of symbols sent. If we define $S$ as the number of FBMC-OQAM symbols (complex field domain), we have:

$\eta_{\text {FBMC-OQAM }}=\frac{S}{K+S-\frac{1}{2}} \eta$.

We can see that as $S$ gets larger, $\eta_{\text {FBMC }}$ tends towards $\eta$. The case of FBMC-QAM is quite similar; as offset QAM is not used, the SE is given by:

$\eta_{F B M C-Q A M}=\frac{S}{K+S-1} \eta$

where $S$ stands for the number of FBMC-QAM symbols. To fairly compare the waveforms, we compute the number of bits that can be transmitted by the waveform given a modulation efficiency $\eta$ and a transmission time from 0.1 to $100 \mathrm{~ms}$. This number of bits is then divided by the number of bits transmitted by an OFDM waveform (or UFMC). The results are depicted in Fig. 10 assuming a sampling frequency of $15.36 \mathrm{MHz}$. UFMC has the same SE as OFDM since the filter length has been defined under this constraint. We can see that as the transmission time gets longer, the SE of FBMC modulations outperforms the SE of OFDM. The efficiency ratio tends to $1+9 / 128$ due to the lack of cyclic prefix. These results clearly demonstrate that the use of $N=1024$ and $K=$ 4 is not well adapted to short transmission time. With this set of parameters, the efficiency ratio is greater than 1 when the transmission time is greater than $2.567 \mathrm{~ms}$ for FBMC-OQAM and $2.06 \mathrm{~ms}$ for FBMC-QAM. Results nevertheless demonstrate that the SE can be increased for short transmission times by decreasing $K$; an efficiency ratio higher than 1 can for example be achieved for a transmission time of $0.2 \mathrm{~ms}$ with appropriate parametrization. However, when $K$ is decreased, the OOB radiation is also increased, making the waveform less robust to relaxed synchronization scenarios (see Section 4). Another degree of freedom is the duration of the symbol. One can imagine to increase the inter carrier spacing (i.e. decrease the FFT size $N$ ); in that case, $K=4$ ensures good frequency localization and the set of parameters is compatible with short transmission time (Fig. 10 bottom, $N=256$ ). The efficiency ratio is greater than 1 with a slot duration greater than $0.2 \mathrm{~ms}$. When the inter carrier spacing is increased, the robustness of the waveform to frequency selective channel is reduced. This effect will be discussed hereafter (Section 3.4) based on simulation results obtained with typical channel models.

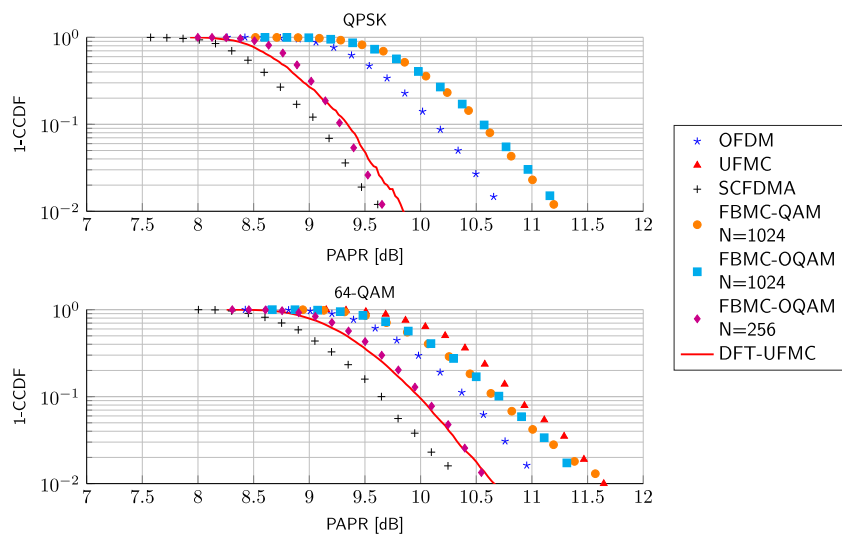

Fig. 11. Comparison of PAPR for different waveforms using QPSK (top) and 64-QAM (bottom).

\subsection{PAPR}

In this section, we compare the PAPR of the different waveforms. The PAPR is defined as the ratio between the peak of squared amplitude and the average power; measured on a burst $b$ :

$\operatorname{PAPR}[b]=\frac{\max \left(\left|y_{b}[k]\right|^{2}\right)}{E\left[\left|y_{b}[k]\right|^{2}\right]}$.

For the statistical analysis, we compute the Complementary Cumulative Density probability Function (CCDF) defined as

$C C D F_{P A P R}(\gamma)=\operatorname{Pr}_{[b]}(P A P R[b]>\gamma)$.

Results are depicted in Fig. 11 for QPSK and 64-QAM. SC-FDMA, due to its (quasi) single carrier property, offers the lowest PAPR. For comparison, the performance when single carrier mode is applied on UFMC (the so-called DFT-UFMC) has been added: SC-FDMA and DFT-UFMC have closed PAPR level. When changing the modulation order from 2 bits per symbol (QPSK) to 6 bits per symbol (64-QAM), PAPR is only slightly affected. Multicarrier waveforms (except SCFDMA), have a comparable PAPR, $2 \mathrm{~dB}$ higher than SC-FDMA. It is well known that the higher the FFT size, the higher the PAPR. As a consequence, for FBMC-OQAM, PAPR is reduced when switching from a 1024-FFT size to a 256-FFT size, leading to almost the same performance as we get for SC-FDMA. This result is interesting for the definition of a waveform dedicated to UL, where a low PAPR is a stringent requirement. Note that in case of OFDM/UFMC and SCFDMA it is also possible to reduce the size of the FFT (i.e. increase the inter carrier spacing) to lower the PAPR. However, for these waveforms and assuming a one tap receiver for channel equalization, performance decrease on fading channel. This aspect will be discussed in the next section.

\subsection{Performance on typical channels}

The BER performance of the waveforms studied in this work have been assessed for two channel models: Extended Vehicular A model (EVA) and Extended Typical Urban model (ETU) [24]. These delay profiles represent a medium and a high delay spread environment, respectively. The multipath delay profiles for these channels are given in Table 1. The parameters used are summarized in Table 2. Perfect channel state information and perfect synchronization are assumed. A max log approximation is used for the demapping and a soft input Viterbi algorithm is implemented.

Fig. 12 first shows the BER for a 64-QAM modulation over ETU channel. Here $N=1024$ for all waveforms. Two DFT sizes are assessed for SC-FDMA, 12 and 24. Three different implementations of FBMC, with $K=4$, are tested: FBMC-OQAM with PPN 
Table 1

Channel delay profile.

\begin{tabular}{|c|c|c|c|}
\hline \multicolumn{2}{|l|}{ EVA } & \multicolumn{2}{|l|}{ ETU } \\
\hline $\begin{array}{l}\text { Excess tap delay } \\
\text { [samples] }\end{array}$ & $\begin{array}{l}\text { Relative power } \\
{[\mathrm{dB}]}\end{array}$ & $\begin{array}{l}\text { Excess tap delay } \\
\text { [samples] }\end{array}$ & $\begin{array}{l}\text { Relative power } \\
{[\mathrm{dB}]}\end{array}$ \\
\hline 0 & +0.0 & 0 & -1.0 \\
\hline 1 & -1.5 & 1 & -1.0 \\
\hline 3 & -1.4 & 2 & -1.0 \\
\hline 5 & -3.6 & 3 & +0.0 \\
\hline 6 & -0.6 & 4 & +0.0 \\
\hline 11 & -9.1 & 8 & +0.0 \\
\hline 17 & -7.0 & 25 & -3.0 \\
\hline 27 & -12.0 & 35 & -5.0 \\
\hline 39 & -16.9 & 77 & -7.0 \\
\hline
\end{tabular}

Table 2

Systems parameters.

\begin{tabular}{|c|c|c|c|}
\hline & & QPSK & 64-QAM \\
\hline \multicolumn{2}{|l|}{ Payload (bits) } & 11192 & 33592 \\
\hline \multicolumn{2}{|l|}{ FEC } & \multicolumn{2}{|c|}{$\mathrm{CC} \mathrm{R}=2 / 3+$ Interleaver } \\
\hline \multicolumn{2}{|l|}{$F_{S}$} & \multicolumn{2}{|c|}{$15.36 \mathrm{MHz}$} \\
\hline \multicolumn{2}{|l|}{ Bandwidth } & \multicolumn{2}{|c|}{$9 \mathrm{MHz}$} \\
\hline \multirow[t]{5}{*}{ Filtering } & OFDM & \multicolumn{2}{|c|}{ NA } \\
\hline & SC-FDMA & \multicolumn{2}{|c|}{ DFT spread } \\
\hline & UFMC & \multicolumn{2}{|c|}{ Dolph-Chebyshev, $L=73$} \\
\hline & FBMC-QAM & \multicolumn{2}{|c|}{ PHYDYAS $K=4$} \\
\hline & FBMC-OQAM & \multicolumn{2}{|c|}{ PHYDYAS $K=4$} \\
\hline \multirow[t]{7}{*}{$N_{a} / N_{F F T}$} & OFDM & \multicolumn{2}{|c|}{$600 / 1024$} \\
\hline & SC-FDMA & \multicolumn{2}{|c|}{$600 / 1024$} \\
\hline & UFMC & \multicolumn{2}{|c|}{$600 / 1024$} \\
\hline & FBMC-QAM & \multicolumn{2}{|c|}{$600 / 1024$} \\
\hline & FBMC-OQAM & \multicolumn{2}{|c|}{$600 / 1024$} \\
\hline & & \multicolumn{2}{|c|}{$300 / 512$} \\
\hline & & \multicolumn{2}{|c|}{$150 / 256$} \\
\hline \multirow[t]{7}{*}{ Packet duration (ms) } & OFDM & \multicolumn{2}{|c|}{0.999} \\
\hline & SCFDMA & \multicolumn{2}{|c|}{0.999} \\
\hline & UFMC & \multicolumn{2}{|c|}{0.999} \\
\hline & FBMC-QAM & \multicolumn{2}{|c|}{1.133} \\
\hline & FBMC-OQAM & \multirow{3}{*}{\multicolumn{2}{|c|}{$\begin{array}{c}1.167\left(N_{F F T}=1024\right) \\
1.050\left(N_{F F T}=512\right) \\
0.992\left(N_{F T}=256\right)\end{array}$}} \\
\hline & & & \\
\hline & & $0.992\left(N_{F F T}=256\right)$ & \\
\hline
\end{tabular}

receiver, FBMC-OQAM and FBMC-QAM with FS receivers. It must first be noted that two waveforms experience a quite high error floor: FBMC-QAM and UFMC. The FBMC-QAM waveform suffers from the non-contiguous nature of the signal (transient effect between interleaved blocks); the complex orthogonality is broken by the channel. This effect introduces self-interference that becomes dominant when the SNR increases. Concerning UFMC, this modulation suffers from the lack of $\mathrm{CP}$ over such a high delay spread channel. SC-FDMA, despite the use of a $\mathrm{CP}$, also presents poor performance: the IDFT spreading at the receiver indeed spreads the high fading occurrences over several carriers. This is all the more true when the DFT is realized on a larger number of carriers. Thanks to the 72 samples long CP, CP-OFDM has good performance. Both implementations of FBMC-OQAM nevertheless outperform CP-OFDM, due to the important length of the filter in the time domain, compared to the channel spreading. FS receiver is the best FBMC-OQAM receiver; it benefits from its high frequency granularity in such a frequency selective channel.

Fig. 13 (for QPSK) and Fig. 14 (for 64-QAM) assess the performance of FBMC-OQAM with different FFT sizes, over the ETU channel. Decreasing the FFT size $N$ for FBMC allows to decrease in the same proportions the length of the filter in the time domain and thereby the length of the FBMC frame (at the expense of a poorer frequency localization). In case of PPN receiver scheme, performance are degraded as the FFT size is reduced. One must

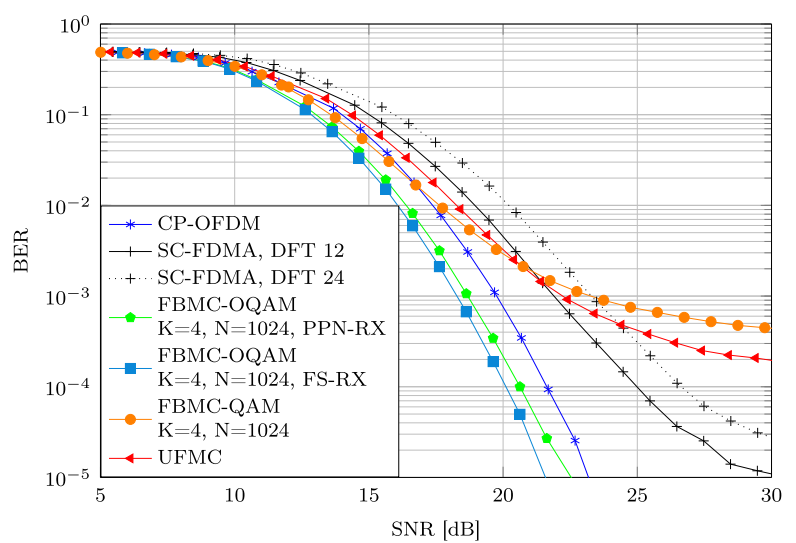

Fig. 12. Performance over ETU channel for 64-QAM.

nevertheless remember that FBMC FS equalizer has a $K$ times higher frequency resolution. For FBMC with $N=256$ and 64-QAM, an error floor appears at $10^{-5}$ when FS scheme is considered for the receiver. For this channel model, the PPN based receiver is not able to decode the signal (error floor at $10^{-1}$ ).

On Figs. 13 and 14, CP-OFDM, SC-FDMA (DFT 12) and UFMC are also plotted for comparison purpose, but with 1024 carriers for all sub-figures. For a QPSK modulation, UFMC does not experience an error floor anymore and perform the same than CP-OFDM. SCFDMA is around $3 \mathrm{~dB}$ worse (at BER $=10^{-5}$ ). With $N=1024$, both PPN and FS receivers schemes perform the same. The frequency resolution of the equalizer is high enough to cope with the frequency selectivity of the channel. When decreasing the FFT size from 1024 to 512, performance of both PPN and FS receivers are similar to performance of CP-OFDM and UFMC receivers. It means that a FFT size of 512 carriers is a high enough resolution for FBMC with a PPN scheme receiver. A significant performance degradation can be noted when $N=256$, for FBMC with a PPN scheme receiver. Nevertheless, the decrease of performance is lower for FBMC FS scheme that takes advantage of the better frequency resolution of the equalization.

On Fig. 14, for the three sub-figures, the curves for CP-OFDM, SC-FDMA and UFMC are the same than on Fig. 12. Here the behavior of both FBMC receivers can be explained in the same way than for Fig. 13, with the substantial difference that FBMC PPN receiver experiences a high error floor if $N<1024$.

The performance of all receivers over EVA channel are depicted in Fig. 15 with 64-QAM modulation. Here, CP-OFDM, SC-FDMA and UFMC are still implemented with a FFT size of 1024, whereas different configurations of FBMC are assessed. In this moderately frequency selective channel, CP-OFDM and UFMC perform the same. Even though the channel is less selective, FBMC-OQAM PPN with $N=256$ still presents an error floor. This is no longer true when the size of FFT is increased to 512 . Unlike in the selective ETU channel scenario, FBMC-QAM with $N=1024$ exhibits worse BER compared to other waveforms. However this effect can be managed by introducing a smoothing filter at the price of an introduction of self-interference. Finally, as expected, FBMC FS receiver performs well, better than CP-OFDM and UFMC, even with a low number of carriers.

Concerning complexity, the authors of [25] concluded that the complexity of a filtered-OFDM transmitter could be as high as or even higher than - the one of a FBMC transmitter, which does not require this additional filtering. Similarly, the complexity of a FBMC receiver has been compared to the one of an OFDM receiver. In [26], it has been shown that the computational complexity of the FS-FBMC receiver was only around 30\% higher than that of the 

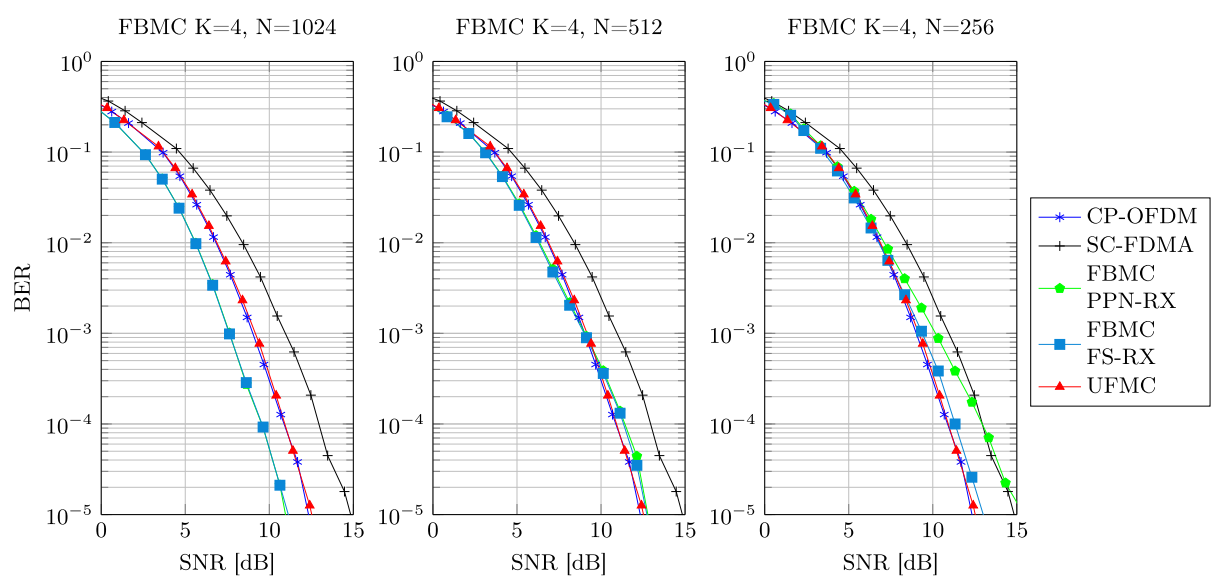

Fig. 13. Performance over ETU channel for QPSK.
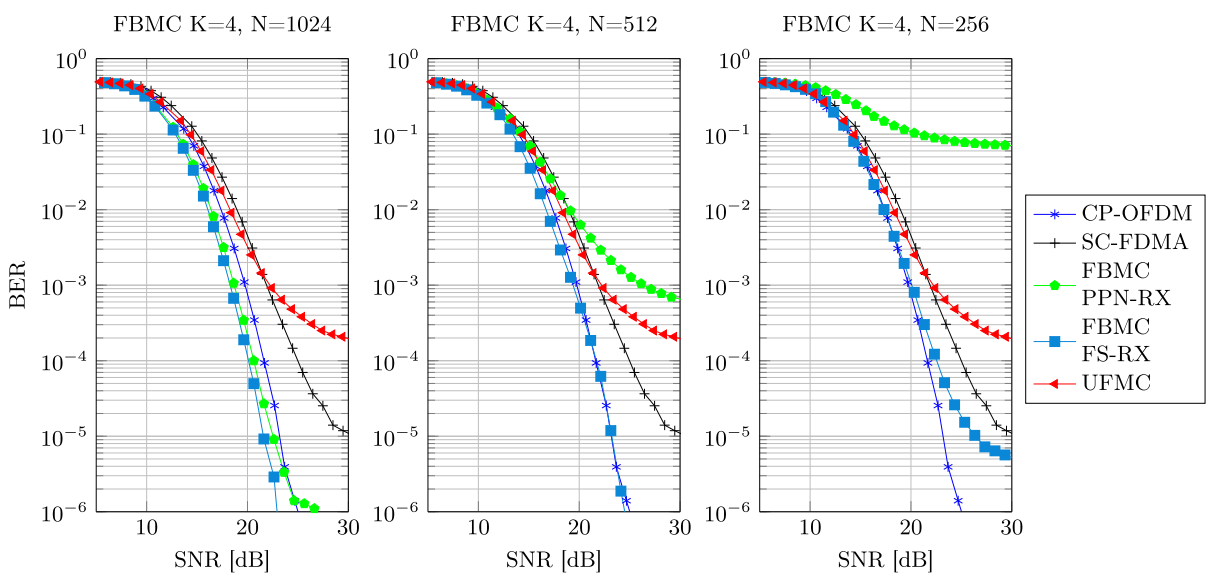

Fig. 14. Performance over ETU channel for 64-QAM.

OFDM receiver, based on FPGA complexity evaluation. In addition, the block-wise processing approach requires FBMC symbols to be stored, which impacts the size of the memory (2.5x the one of an equivalent $\mathrm{CP}-\mathrm{OFDM}$ receiver). However, such memory sizes can be implemented at a very limited footprint and cost on current silicon technology nodes.

\section{Robustness to relaxed synchronization uplink scenario}

As stated in the introduction, multi-user asynchronous access scenario is of importance in the next $5 \mathrm{G}$ due to the massive number of expected devices. In the current standardization process, performance of the waveform on relaxed synchronization scenarios are discussed [27]. In this section, we analyze the multi-user asynchronous access scheme in terms of inter-user interference for the different investigated waveforms. We then use these results to solve a bit loading optimization problem. We first introduce the scope of this scenario with the presentation of the unified frame structure scenario.

\subsection{Scenario: unified frame structure}

Flexible and efficient use of all available non-contiguous spectra for widely different network deployment scenarios is one challenge for the future $5 \mathrm{G}$. To maximize $\mathrm{SE}$, the $5 \mathrm{G}$ air interface technologies will need to be flexible and capable of mapping various services to the best suitable combinations of frequency and time resources. In order to provide a uniform service experience to

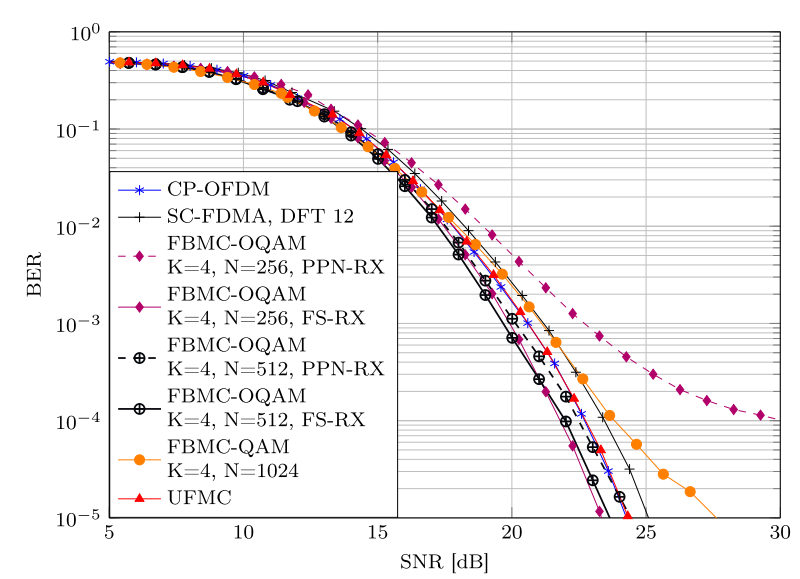

Fig. 15. Performance over EVA channel for 64-QAM.

users with the premises of heterogeneous networking, the authors of [2] have introduced the concept of a unified frame structure for $5 \mathrm{G}$. The idea is to provide a flexible multi-service solution in an integrated air interface. A frame, divided into different areas of services, has been proposed with different Time Transmission Interval (TTI) durations. These services include high data rate and/or low latency traffic (short TTI), scheduled and non-synchronous traffic. Scheduled transmissions, as already in place in LTE and LTE$\mathrm{A}$, are dedicated to high bandwidth data pipes and Machine Type 


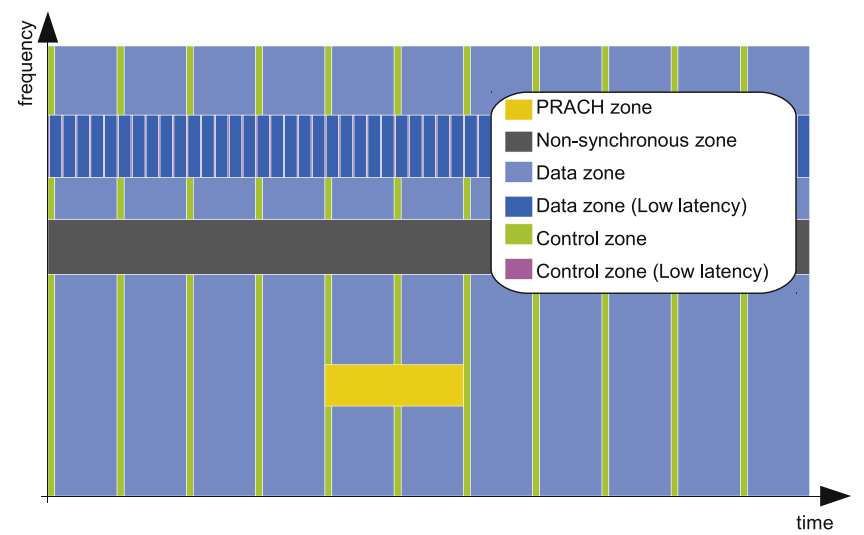

Fig. 16. Example of $5 \mathrm{G}$ unified frame structure. (For interpretation of the references to color in this figure legend, the reader is referred to the web version of this article.)

Communications (MTC) with Quality of Service (QoS) control. Sporadic traffic uses non-scheduled contention based access methods designed to efficiently enable MTC type payloads, as well as to bring an efficient solution to the fast dormancy issue.

A possible example of the time-frequency grid is shown in Fig. 16. The grid is divided into six zones. The Physical Random Access Channel (PRACH) zone (yellow zone), as already in place in LTE, is dedicated to the UE timing synchronization process and is used to obtain the RBs (e.g. connection request). This random access channel is dedicated to the initialization of strictly synchronized/scheduled transmission. Data zone (blue) and Control zone (green) are dedicated to normal traffic (e.g. LTE typical use-cases). A control zone starts, followed by the data zone. Control zone carries control information such as RB allocation information, modulation and coding scheme information, etc. A low latency zone is also depicted (dark blue and magenta). In that case the TTI is shorter. The structure with a control zone followed by the data zone is maintained. Tightly scheduled and network controlled resource allocation based traffics are examples that could be mapped on low latency zone. The TTI of the normal zone is an integer multiple of the one defined for the low latency zone. Finally, a nonsynchronous zone is introduced to deal with non-synchronous transmission. This zone is dedicated to sporadic traffic that uses contention-like based approaches with random access designed to efficiently enable MTC type payloads and bring an efficient solution to the fast dormancy issue. Of course, to keep the flexibility needed by the $5 \mathrm{G}$ requirements, dynamic adaption of the zones should be possible. The $5 \mathrm{G}$ waveforms described in Section 2 may impact the unified frame structure presented above. In particular OQAM signaling implies the redesign of classical LTE reference symbols for synchronization, channel estimation and cell search mechanism. Furthermore, waveforms that are not well localized in time, such as FBMC, may be seen at a first glance less suitable to short packet transmissions and low latency zones. This assertion must be balanced since the pulse duration of FBMC can be lowered by decreasing the FFT size. Furthermore, it also depends on how is defined the TTI. Indeed if the TTI is defined as the duration of the window on which the FFT is applied at the receiver side, or in other words if the TTI does not take into account the time spread of the pulse shaping function as well as the overlapping as in $3 \mathrm{G}$ (i.e. the overlapping symbols with FBMC in this case), then there is no spectral efficiency loss with FBMC especially for short TTIs.

In order to be efficient, this structure clearly demands to revisit the strict synchronicity and orthogonality that both prevail in current LTE-A systems. This new requirement leads to reconsider the transmission technique and consequently the transceiver structure of the 5 th generation of cellular networks.

\subsection{System model}

Based on the frame structure described hereinbefore, a simplified model for UL is derived where all the zones use the same waveform contender. A set of three users is simulated as illustrated in Fig. 17. We assume perfect synchronization with the serving BS and perfect channel estimation of the user of interest, User Equipment (UE) $0 . N_{a}=12$ carriers (or 1 resource block) are allocated to UE0, and 36 carriers for UE1 and UE2. UE1 and UE2 are supposed not synchronized with UE0 (and thus not synchronized with the BS). The inter-carrier spacing is set to $15 \mathrm{kHz}$, the FFT size $N=1024$, and for OFDM waveforms, a Guard Interval (GI) of 72 samples (approximately $1 / 14 \times N$ ) is assumed. These parameters have been inspired by possible extensions of LTE parameters. The UE using the non-synchronous zone is not time synchronized with the other zones. Due to the timing and frequency errors, the nonsynchronous zone is corrupted by interference generated by the adjacent zones. One could imagine that UEs send their data in the non-synchronous zone after a frequency synchronization that entitles the UE to know the localization of the non-synchronous zone in the time frequency grid.

\subsection{Simulation results}

We consider the parameters described in Table 3. To quantify the influence of the interference, neither noise nor channel effect are added. Contrary to the authors of [22,28], we propose to measure the MSE in $\mathrm{dB}$, per carrier, versus the delay error. The MSE is calculated at the receiver side, on the equalized constellation. The delay error is defined as the time offset between UE0 time of arrival and UE1 time of arrival (which is identical to UE2 time of arrival, as these 2 users are synchronized). The use of the MSE per carrier (instead of a mean MSE) is of importance as the interference is not uniform across the carriers. As discussed later, it also gives strong hints to optimize the performance of the transmission. Therefore, we depict in Fig. 18 the MSE for the user of interest, per carrier, versus the delay error for OFDM, SC-FDMA, UFMC (with and without additional windowing at the receiver) and FBMC (with an IFFT/FFT size of 1024 and 256). The computed MSE is a two dimensional matrix: one dimension is related to the carrier index $i$ and the other dimension corresponds to the delay error $n$. For each couple $(i, n)$ the obtained MSE is linked to a color: from red ( high interference level) to blue ( no interference). The displayed MSE per carrier gives information about the interference level but also how interference spreads in the frequency plane. We also consider several Guard Carriers (GC) between the two zones: 0 GC (contiguous allocation), 1 GC and 2 GCs. For FBMC with an IFFT/FFT size of 256, the guard space and the user bandwidth in $\mathrm{Hz}$ are the same as for the other waveforms based on 1024 IFFT/FFT size. The effective frequency spacing between the two zones is thus lower than a GC for FBMC256. In all the cases, we assume that the received signals from the non-synchronous zone have the same power as the signals from other zones.

As expected, the interference level is strong for OFDM and SCFDMA, especially when there is no GC. The orthogonality is though preserved if the delay error is shorter than the $\mathrm{CP}$ duration. For OFDM, the interference level decreases steadily with the subcarrier index, from the interferer, whereas for SC-FDMA, the DFT operation spreads the interference on all the RB. UFMC shows better performance compared to OFDM, which is consistent with [22]. The effect of the windowing process is clear, especially when the carriers are far from the interferer. For FBMC, due to the good spectral location, only one carrier is affected by the interference. This is a direct consequence of a built-in property of the FBMC waveform. The prototype filter has been designed to minimize OOB interference and only the carriers located at the edges of 


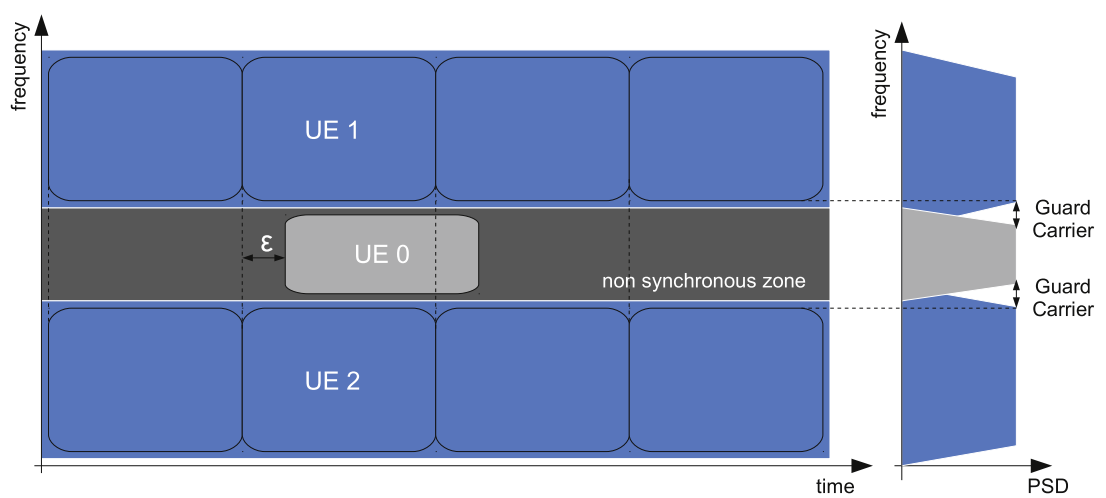

Fig. 17. Proposed simplified scenario.

Table 3

Simulation parameters

\begin{tabular}{ll}
\hline Common parameters & \\
\hline Modulation scheme & QPSK \\
Allocated Carriers for "non synchronous zone" & $12(3$ for FBMC-256) \\
Allocated Carriers for "adjacent zone" & $3 \times 12(9$ for FBMC-256) \\
Sampling frequency & $15.36 \mathrm{MHz}$ \\
Power offset between zones & $0 \mathrm{~dB}$ \\
\hline OFDM and SC-FDMA & \\
\hline Cyclic prefix & 72 samples \\
DFT size & 12 \\
\hline UFMC & \\
\hline Filter length & 73 \\
Stop band attenuation (dB) & 40 dB \\
Windowing & Raised cosine \\
\hline FBMC & \\
\hline$K$ & 2,4 \\
Prototype Filter & PHYDYAS [5] \\
\hline
\end{tabular}

the spectrum are dominated by the interference. This is because the orthogonality of FBMC-OQAM adjacent users is not preserved when phase coherence is not guaranteed between zones. When at least one GC is inserted there is no interference for FBMC-1024, and the interference for FBMC-256 becomes very low. Besides, there is no interference if we consider a frequency spacing of $30 \mathrm{kHz}$ (which means half a guard carrier for FBMC-256). FBMC shows a better behavior for multi-user access scheme, especially when one GC is inserted. For FBMC-1024, it should also be noted that negligible interference error can be reached with the insertion of half a guard carrier, which leads to a better SE. If we use a FBMC-OQAM signal with an overlapping factor $K=2$, a loss of performance is depicted: the interference level is stronger for the two carriers at the edges of the bandwidth. If we consider FBMC-QAM, the interference level is strong for the even carriers: this is due to the block-wise permutation that introduces additional interference when secondary users interfere with the user of interest.

\subsection{Optimization of the spectral efficiency}

As the interference level varies from one carrier to another, optimizing the SE by considering adaptive modulation, or bit loading scheme, for the non-synchronous zone can be envisaged. Conventional multicarrier systems use a fixed constellation on all carriers. Thus, the total BER is dominated by the carriers that have worst performance (carriers at the border of the fragment for OFDM, UFMC and FBMC without GCs). To improve the system performance, adaptive bit allocation can be employed such that the information is redistributed across the carriers, according to the a priori knowledge of the interference. Contrary to classical bit loading scheme, the allocation in our case should be static as one shot transmission is assumed. As the UEs do not have the knowledge of the timing misalignment, we propose to compute an a priori bit allocation assuming a given average level of interference under the assumption that the power of the signal received in the non-synchronous zone is close to the power of the adjacent zones.

For a multicarrier modulation, noise caused by interference generated when timing misalignment occurs at the receiver adds to thermal noise. Assuming that interference noise caused by misalignment of the receiver and thermal noise are independent, the total noise variance per active carrier may be written as:

$\sigma^{2}(k)=\sigma_{n}^{2}+\sigma_{i}^{2}(k)$

where $\sigma_{n}$ is the standard deviation of the thermal noise and $\sigma_{i}(k)$ is the standard deviation of the interference generated on the $k$ th active carrier. $\left(\sigma_{i}(k)\right.$ also depends on the delay error as discussed in the previous subsection.)

Based on these assumptions, a bit loading optimization problem can be stated as follows: the classical bit allocation problem requires the knowledge of the interference level for each time misalignment. In our proposed work, we assume that this information is not available, but we know that we have to cope with interference from non-synchronous adjacent UE. Therefore, we propose to average the interference level on a carrier over all the possible timing misalignments. This level of interference is used to solve the bit allocation problem

$$
\eta=\underset{\substack{\eta_{0}, \ldots, \eta_{N a-1} \in S \\ P e\left[\eta_{0}, \ldots, \eta_{N a-1}\right]<\Gamma}}{\operatorname{argmax}}\left\{\frac{1}{N_{a}} \sum_{k=0}^{N_{a}-1} \eta_{k}\right\}
$$

where $N_{a}$ is the number of active carriers, $\eta_{k}$ is the number of bits transmitted by subcarrier $k, \Gamma$ is a BER threshold, $S$ is the subset of the supported bit per symbol indexes, and $P_{e}\left(\eta_{0}, \ldots, \eta_{N a-1}\right)$ is defined as:

$P_{e}\left(\eta_{0}, \ldots, \eta_{N a-1}\right)=\frac{\sum_{k=0}^{N_{a}-1} \eta_{k} P\left(\eta_{k}, \sigma^{2}(k)\right)}{\sum_{k=0}^{N_{a}-1} \eta_{k}}$

where $P\left(\eta_{k}, \sigma^{2}(k)\right)$ is the BER for subcarrier $k . P_{e}\left(\eta_{0}, \ldots, \eta_{N a-1}\right)$ corresponds to a mean bit error rate measure w.r.t the modulation scheme applied to each subcarrier. This optimization problem tries to maximize the SE under the constraint of a given BER. This optimization makes sense only if the magnitude of the channel coefficients seen by each carrier are close to each other (block fading). This assumption is true if the bandwidth is limited to one RB.

The optimization result is depicted in Fig. 19 for $\Gamma=10^{-3}$ (uncoded BER). First, it is shown that UFMC SE is the same as the OFDM SE for low SNR, and outperforms OFDM SE if the SNR is higher than 

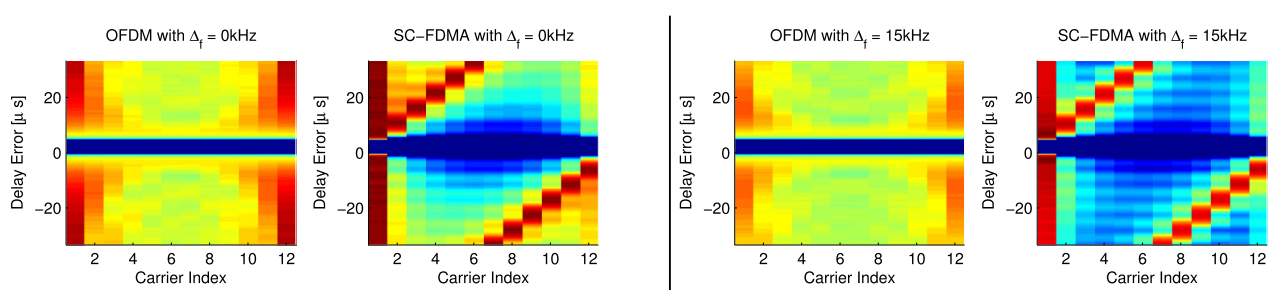

UFMC with $\Delta_{\mathrm{f}}=0 \mathrm{kHz}$

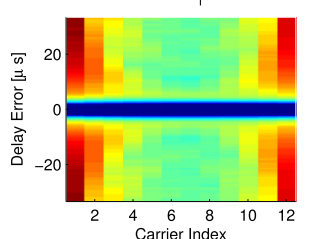

wUFMC with $\Delta_{\mathrm{f}}=0 \mathrm{kHz}$

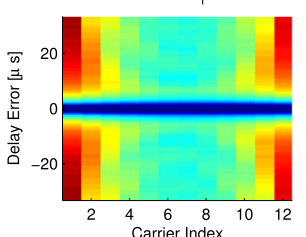

UFMC with $\Delta_{\mathrm{t}}=15 \mathrm{kHz}$

wUFMC with $\Delta_{\mathrm{f}}=15 \mathrm{kHz}$

FBMC with $\Delta_{\mathrm{f}}=\mathrm{OkHz}$

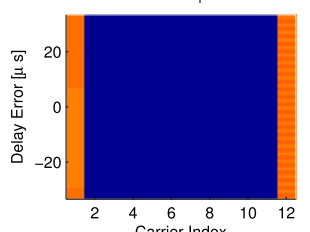

FBMC-256 with $\Delta_{\mathrm{f}}=\mathrm{OkHz}$

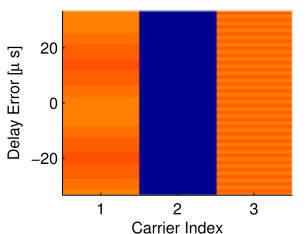

FBMC, $K=2$ with $\Delta_{\mathrm{f}}=0 \mathrm{kHz}$

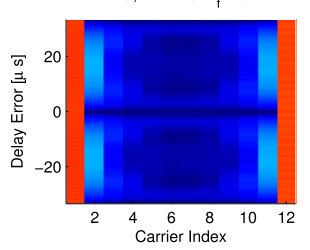
FBMC-QAM with $\Delta_{\mathrm{f}}=0 \mathrm{kHz}$
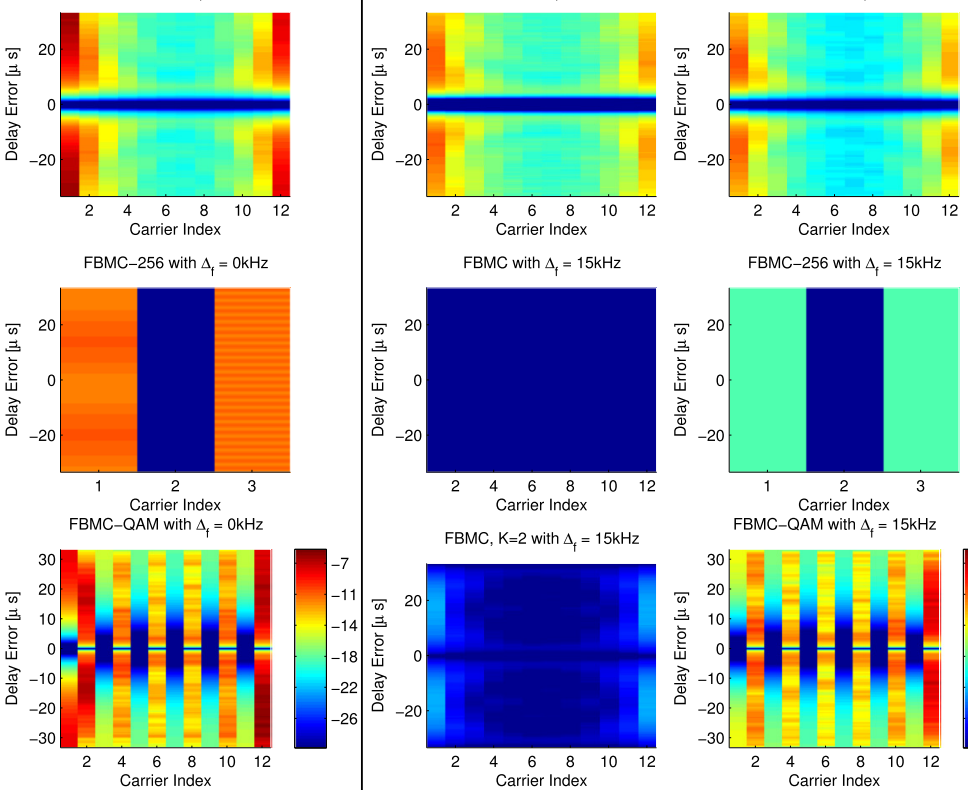

FBMC with $\Delta_{\mathrm{f}}=15 \mathrm{kHz}$

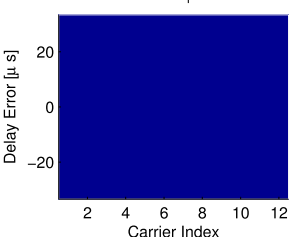

FBMC-256 with $\Delta_{\mathrm{f}}=15 \mathrm{kHz}$

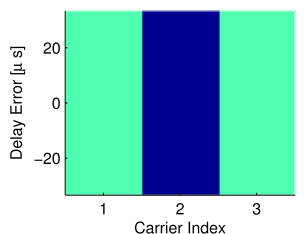

FBMC, $K=2$ with $\Delta_{f}=15 \mathrm{kHz}$
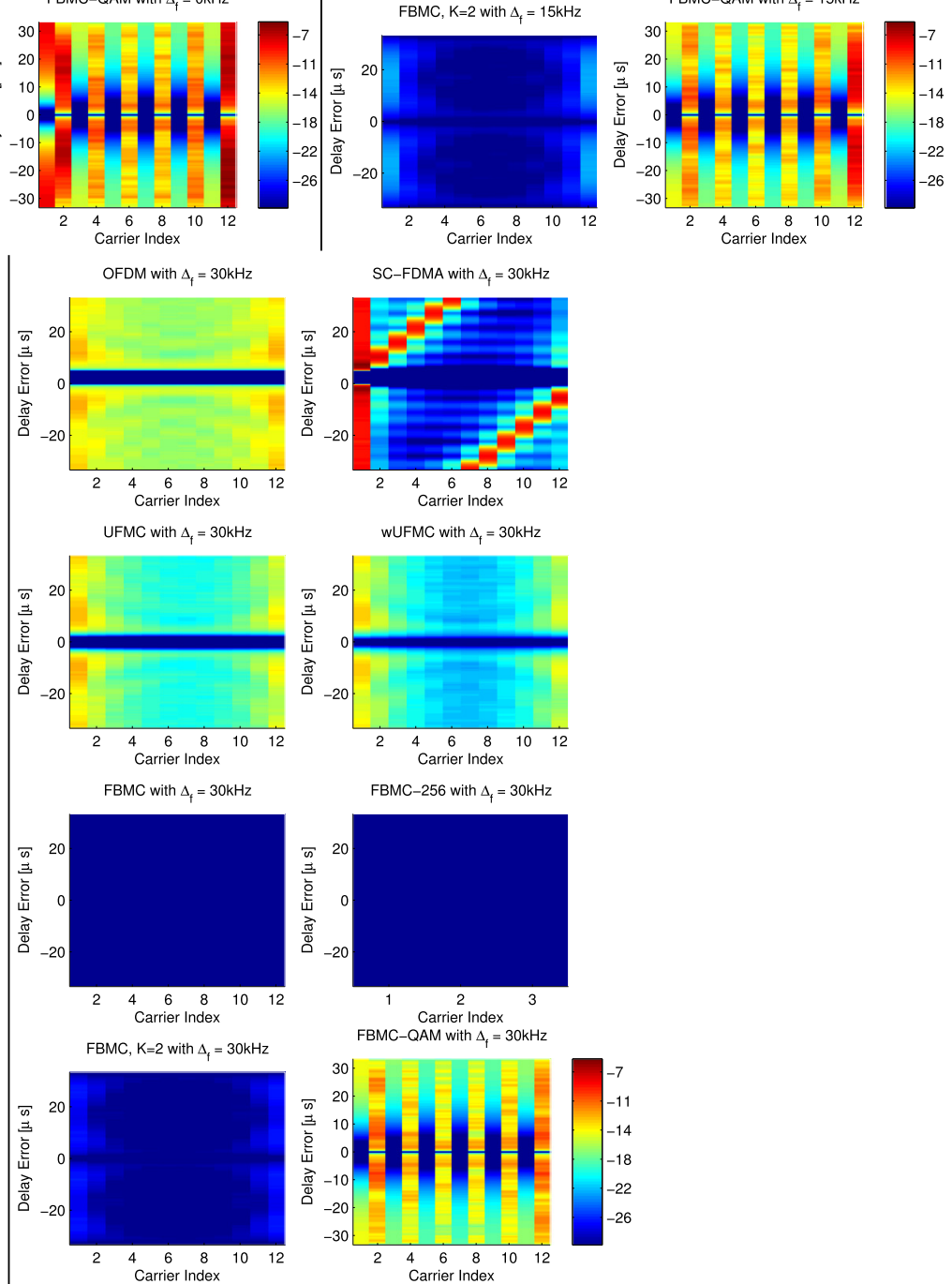

FBMC-256 with $\Delta_{\mathrm{f}}=30 \mathrm{kHz}$

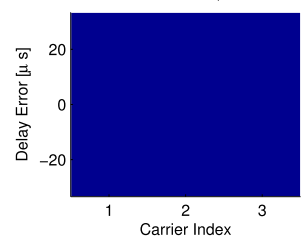

FBMC-QAM with $\Delta_{\mathrm{f}}=30 \mathrm{kHz}$

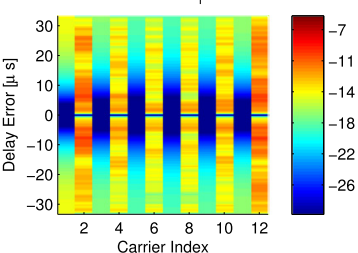

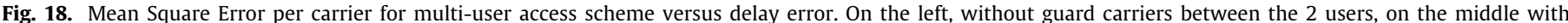
$\Delta_{f}=15 \mathrm{kHz}$ (one GC), and on the right with $\Delta_{f}=30 \mathrm{kHz}$ (two GCs). 


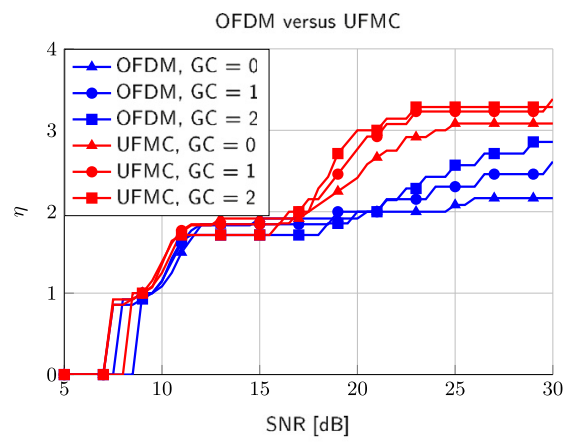

OFDM versus SCFDMA

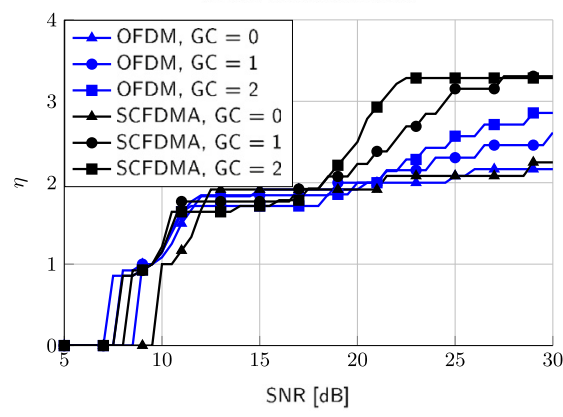

OFDM versus $F B M C-1024 \mathrm{~K}=4$

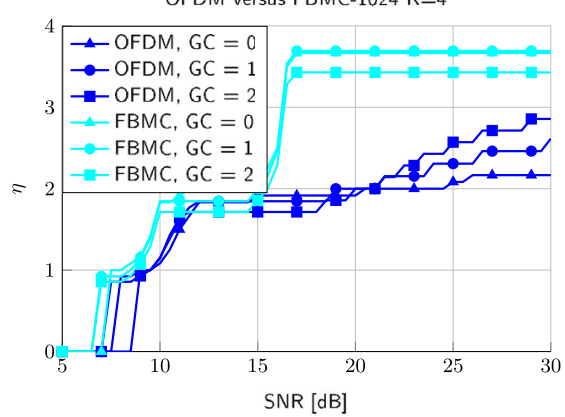

FBMC $K=4$ versus $F B M C K=2$

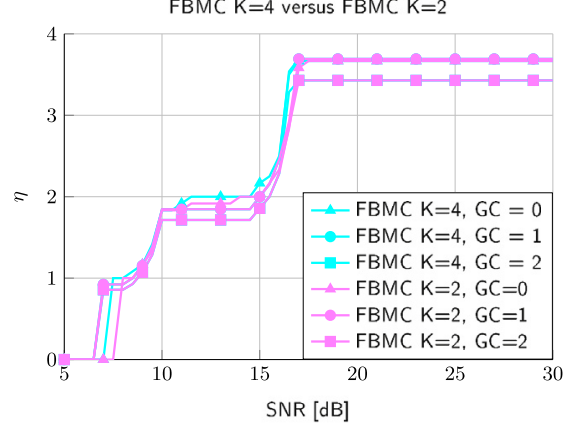

FBMC-256 $K=4$ versus UFMC

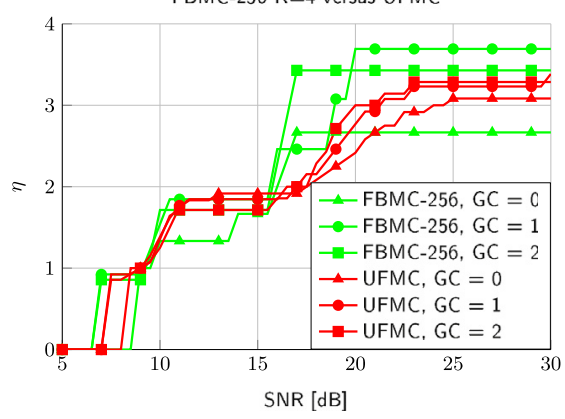

wUFMC versus UFMC

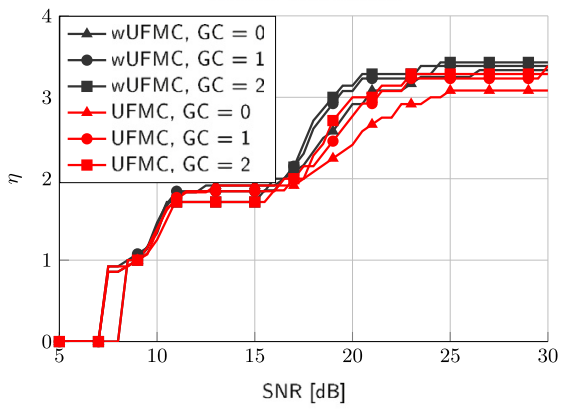

SCFDMA versus UFMC

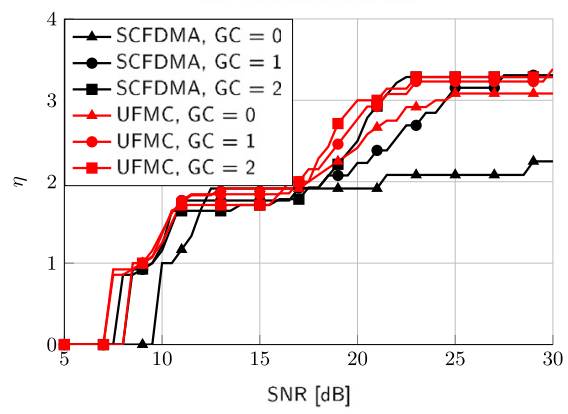

FBMC-1024 $\mathrm{K}=4$ versus UFMC

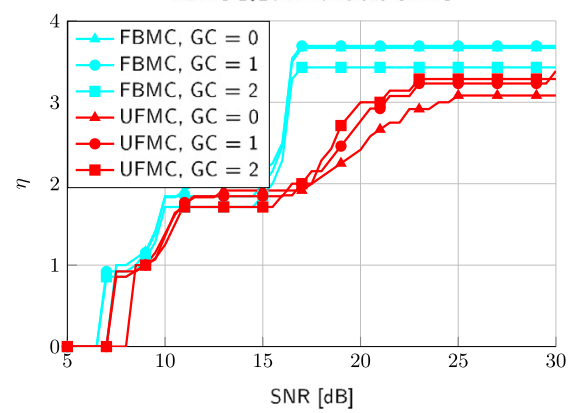

FBMC-1024 versus FBMC-256

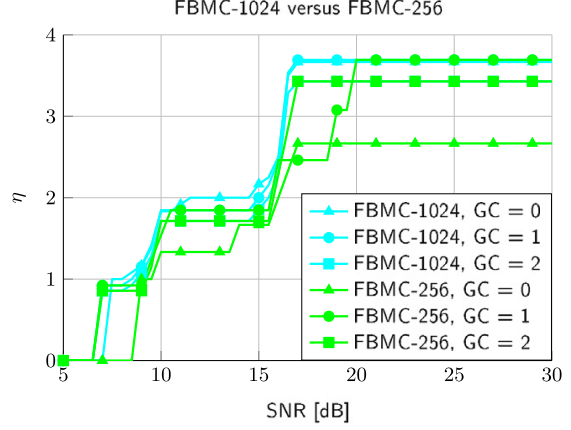

Fig. 19. Bit loading optimization $\{$ BPSK, QPSK,16-QAM $\}$ for $5 \mathrm{G}$ candidates versus channel SNR for $\Gamma=10^{-3}$. $y$ axis represents $\eta$, the average SE per carrier.

$16 \mathrm{~dB}$. Besides, despite the SE loss introduced by the insertion of GCs, the UFMC SE still increases with the increase of the number of GCs. The maximal SE is obtained with the insertion of $2 \mathrm{GCs}$. It is also noticeable that, with high SNR, UFMC SE is always higher than OFDM SE, even when no GC is inserted for UFMC and 2 GCs are inserted for OFDM. Similar results are obtained for bit loading with 
and without the additional windowing process for UFMC. Indeed, windowing process for UFMC improves the performance for the carriers far from the interferer (see Fig. 18) where the modulation order is already high for UFMC (as the interference is limited). The SE gain obtained with the additional windowing is thus limited.

Bit loading optimization result for OFDM and SC-FDMA, without GC, are very comparable. Surprisingly, as soon as at least one GC is inserted, SC-FDMA provides better results than OFDM. This point is particularly interesting as OFDM and SC-FDMA have the same MSE if the measure is done on a complete RB. However, as shown in Fig. 18, the MSE per subcarrier is different, as for SC-FDMA one subcarrier (the left one) is particularly corrupted by the inter-user interference. It also legitimates the use of carrier MSE instead of global MSE to estimate the impact of the inter-user interference. If at least one GC is inserted for UFMC and SC-FDMA, the performance are quite similar, but in the case of no additional GC, UFMC then outperforms SC-FDMA.

FBMC waveform provides better performance compared to OFDM. It is also noticeable that the insertion of a GC does not improve the performance, due to the spectral loss introduced by the use of the GC. This is the reason why the SE is lower for FBMC with $G C=2$ compared to FBMC with $G C=1$ as the insertion of two GCs (with respect to 1) does not change the carrier MSE performance (see Fig. 18) but still lowers the SE. As we consider 12 carriers for the user of interest, the insertion of GC introduces a severe loss of SE that explains the aforementioned result. Finally, we compare the SE between UFMC and FBMC. It is shown that whatever the GC configuration, FBMC has better performance than UFMC. It is also remarkable that even without GC, the overall SE of FBMC outperforms UFMC SE when 2 GC are inserted (which is the best case for UFMC). The initial results for FBMC-OQAM have been stated with a FFT size of 1024 and an overlapping factor $K=4$. These two parameters can be adapted with the reduction of the FFT size (from 1024 to 256) or of the overlapping factor (from 4 to 2). In the first case, it is shown that the performance can be enhanced and optimized with the insertion of a GC. In this case, better performance is obtained, compared to the other multicarrier waveforms (and UFMC in particular). If an overlapping factor of 2 is used, the performance is almost the same as with an overlapping factor of 4.

It should be mentioned that these results are valid as long as the number of active carriers is small. If the number of carriers is increased, the effect of the interference, located at the edge of the spectrum, becomes negligible. However, we believe that in the context of MTC, the assumption of a small number of active carriers makes sense.

\section{Conclusion and further discussions}

Flexible and efficient use of all available non-contiguous spectra for widely different network deployment scenarios are one challenge for the future 5G. To maximize SE, the 5G air interface technologies will need to be flexible and capable of mapping various services to the best suitable combinations of frequency and radio resources. Therefore flexibility and good frequency localization of the waveform are key requirements. Legacy OFDM/SC-FDMA waveforms could be a good candidate if a filtering process is added. However, as the number of possible configurations of radio resource mapping is large, the associated complexity will be very expensive.

In this work, a fair comparison of several 5G waveform candidates (OFDM, SC-FDMA, UFMC, FBMC-OQAM and FBMC-QAM) is proposed under a common framework. PSD, SE, PAPR and robustness to asynchronous multi-user UL transmission are assessed. Based on these results, a bit loading algorithm is proposed to cope with the non-uniform distribution of the interference across the carriers in order to increase the SE. We demonstrate that if an $a$ priori information on the SNR is available, it is possible to highly increase the SE of the transmission.

UFMC waveform is an interesting option. The SE is comparable to the one of OFDM and the pulse shaping function gives robustness to access with relax synchronization compared to OFDM. UFMC also preserves backward compatibility with well-known OFDM algorithms (channel estimation, MIMO detectors). On the other hand, the interest of FBMC-QAM is limited, considering actual literature proposals. However one can imagine future enhancements in the filter design.

FBMC.OQAM goes further. The well localized frequency response entitles the use of fragmented spectrum with minor interference on adjacent bands. Very good performances are demonstrated in non-synchronous access (whatever the time delay between users). However, under the time-frequency localization relationship, the time localization is sacrificed, making this new waveform difficult to adapt to short packet size. It has nevertheless been demonstrated in this paper that increasing the inter carrier spacing could give FBMC very beneficial properties. By this means, PAPR is reduced compared to legacy LTE CP-OFDM (and UFMC), the support of small packet size is possible without large overhead (contrary to the classical $N=1024$ configuration) and performance over frequency selective channel is managed with the adoption of a frequency spreading receiver scheme. A configuration with an inter carrier spacing of $60 \mathrm{kHz}$ and a FFT size of $N=256$ could be very attractive for the next generation of cellular network in the UL and in the Downlink (DL).

On the other hand, the absence of guard period gives FBMC an efficiency gain for larger packet size or broadcast transmission (in the DL for instance). One of the other challenges FBMC waveform is facing is the adaption to multiple antenna schemes. This issue is not straightforward and concepts have to be revisited. Finally, the intrinsic complexity of the transceiver is higher (it is also true for UFMC) compared to OFDM transceivers. To be fair, FBMC transceiver complexity still needs to be compared with the complexity of an OFDM transceiver with embedded digital or analog filtering functions. In that case, when flexibility is required (dynamic spectrum allocation on non-contiguous spectrum), the complexity of FBMC and UFMC transceivers could be few orders of magnitude below. Of course, performance as well as equalization complexity heavily depend on the type of prototype filter. In this work we limit our comparisons to the PHYDYAS prototype filter. Optimization depending on the channel as well as comparison of various prototype filter have been already studied in the literature. The interested readers can refer to [8] or [29]. Finally, performance of the studied waveforms are summarized in a synthesis radar chart in Fig. 20. OFDM is here defined as reference.

The benefits of new waveforms for the $5 \mathrm{G}$ use cases have been clearly highlighted. UFMC offers improvements, while keeping backward compatibility with legacy OFDM, while FBMC-OQAM goes forward, making this waveform particularly interesting for $5 \mathrm{G}$ scenarios. We indeed demonstrated the benefits of the FBMCOQAM waveform compared to legacy LTE waveforms and UFMC in terms of PSD, SE, and robustness against both asynchronicity and high delay spread environment. Last but not least, the parametrization of FBMC-OQAM (with the use of various intercarrier spacing or different overlapping factors) allows better flexibility to cope with various services and scenarios.

For a future deployment, some concepts should nevertheless be revisited such as MIMO schemes for FBMC.

\section{Acknowledgments}

This work has been performed in the framework of the Horizon 2020 project FANTASTIC-5G (ICT-671660) receiving funds from 


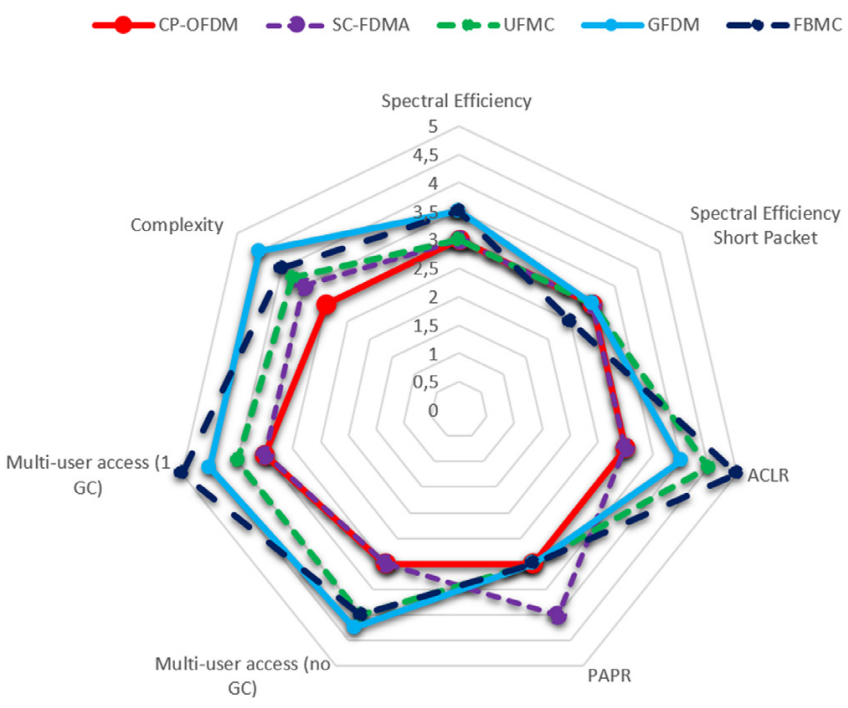

Fig. 20. Waveform comparison: synthesis radar chart.

the European Union and WONG5 project (ANR-15-CE-25-0005-02) receiving funds from the French Research National Agency (ANR). The views expressed in this contribution are those of the authors and do not necessarily represent the projects.

\section{References}

[1] 3GPP RAN 5G Workshop, 2015. http://www.3gpp.org/newsevents/3gppnews/1734-ran_5g.

[2] G. Wunder, P. Jung, M. Kasparick, T. Wild, F. Schaich, Y. Chen, S. Brink, I. Gaspar, N. Michailow, A. Festag, L. Mendes, N. Cassiau, D. Ktenas, M. Dryjanski, S. Pietrzyk, B. Eged, P. Vago, F. Wiedmann, 5GNOW: non-orthogonal, asynchronous waveforms for future mobile applications, IEEE Commun. Mag. 52 (2) (2014) 97-105. http://dx.doi.org/10.1109/MCOM.2014.6736749.

[3] F. Zhao, Huawei smartphone solutions white paper, 2012.

[4] V. Vakilian, T. Wild, F. Schaich, S. ten Brink, J.-F. Frigon, Universal-filtered multi-carrier technique for wireless systems beyond LTE, in: Proc. IEEE Globecom Workshops, GC Wkshps, 2013, pp. 223-228. http://dx.doi.org/10.1109/ GLOCOMW.2013.6824990.

[5] FP7 european project-PHYDYAS: physical layer for dynamic spectrum access and cognitive radio, http://www.phydyas-ict.org.

[6] H. Nam, M. Choi, C. Kim, D. Hong, S. Choi, A new filter-bank multicarrier system for QAM signal transmission and reception, in: Communications, ICC, 2014 IEEE International Conference on, 2014, pp. 5227-5232. http://dx.doi.org/10. 1109/ICC.2014.6884151.

[7] P. Banelli, S. Buzzi, G. Colavolpe, A. Modenini, F. Rusek, A. Ugolini, Modulation formats and waveforms for $5 \mathrm{G}$ networks: Who will be the heir of OFDM?: An overview of alternative modulation schemes for improved spectral efficiency, IEEE Signal Process. Mag. 31 (6) (2014) 80-93. http://dx.doi.org/10.1109/MSP. 2014.2337391.

[8] A. Sahin, I. Guvenc, H. Arslan, A survey on multicarrier communications: prototype filters, lattice structures, and implementation aspects, IEEE Commun. Surv. Tutor. 16 (3) (2014) 1312-1338. http://dx.doi.org/10.1109/SURV.2013. 121213.00263.

[9] B. Farhang-Boroujeny, OFDM versus filter bank multicarrier, IEEE Signal Process. Mag. 28 (3) (2011) 92-112. http://dx.doi.org/10.1109/MSP.2011.940267.

[10] X. Zhang, L. Chen, J. Qiu, J. Abdoli, On the waveform for 5G, IEEE Commun. Mag. 54 (11) (2016) 74-80. http://dx.doi.org/10.1109/MCOM.2016.1600337CM.

[11] A.A. Zaidi, R. Baldemair, H. Tullberg, H. Bjorkegren, L. Sundstrom, J. Medbo, C. Kilinc, I.D. Silva, Waveform and numerology to support 5G services and requirements, IEEE Commun. Mag. 54 (11) (2016) 90-98. http://dx.doi.org/10. 1109/MCOM.2016.1600336CM.

[12] C. Ibars, U. Kumar, H. Niu, H. Jung, S. Pawar, A comparison of waveform candidates for 5G millimeter wave systems, in: 49th Asilomar Conference on Signals, Systems and Computers, 2015, pp. 1747-1751. http://dx.doi.org/10. 1109/ACSSC.2015.7421450.

[13] A.A. Zaidi, J. Luo, R. Gerzaguet, A. Wolfgang, R.J. Weiler, J. Vihriala, T. Svensson, Y. Qi, H. Halbauer, Z. Zhao, P. Zetterberg, H. Miao, A preliminary study on waveform candidates for $5 \mathrm{G}$ mobile radio communications above $6 \mathrm{GHz}$, in: Proc. IEEE 83rd Vehicular Technology Conference, VTC Spring, 2016, pp. 1-6. http://dx.doi.org/10.1109/VTCSpring.2016.7504096.
[14] H. Myung, J. Lim, D. Goodman, Single carrier FDMA for uplink wireless transmission, IEEE Veh. Technol. Mag. 1 (3) (2006) 30-38. http://dx.doi.org/10. 1109/MVT.2006.307304.

[15] M. Bellanger, et al., FBMC physical layer: A primer, 2010. http://www.ictphydyas.org.

[16] M. Bellanger, FS-FBMC: An alternative scheme for filter bank based multicarrier transmission, in: Communications Control and Signal Processing, ISCCSP, 2012 5th International Symposium on, 2012, pp. 1-4. http://dx.doi.org/10. 1109/ISCCSP.2012.6217776.

[17] J.-B. Doré, V. Berg, N. Cassiau, D. Kténas, FBMC receiver for multi-user asynchronous transmission on fragmented spectrum, EURASIP J. (2014) Special issue on advances in flexible multicarrier waveform for future wireless communications.

[18] J.-B. Doré, V. Berg, D. Kténas, Channel estimation techniques for 5G cellular networks: FBMC and multiuser asynchronous fragmented spectrum scenario, Trans. Emerging Telecommun. Technol. (2014).

[19] T. Lee, Y. Ahn, D. Sim, C. Lee, T. Kim, J. Seol, QAM-FBMC system with a robust prototype filter in multipath fading channels, in: Consumer Electronics, ISCE 2014, the 18th IEEE International Symposium on, 2014, pp. 1-2. http://dx.doi. org/10.1109/ISCE.2014.6884502.

[20] X. Wang, T. Wild, F. Schaich, Filter optimization for carrier-frequency- and timing-offset in universal filtered multi-carrier systems, in: Proc. IEEE 81st Vehicular Technology Conference, VTC Spring, 2015, pp. 1-6. http://dx.doi.org/ 10.1109/VTCSpring.2015.7145842.

[21] F. Schaich, T. Wild, Waveform contenders for 5G 2014; OFDM vs. FBMC vs. UFMC, in: 6th International Symposium on Communications, Control and Signal Processing, ISCCSP, 2014, pp. 457-460. http://dx.doi.org/10.1109/ISCCSP. 2014.6877912.

[22] T. Wild, F. Schaich, Y. Chen, 5G air interface design based on Universal Filtered (UF-)OFDM, in: 19th International Conference on Digital Signal Processing, DSP, 2014, pp. 699-704. http://dx.doi.org/10.1109/ICDSP.2014.6900754.

[23] F. Schaich, T. Wild, Y. Chen, Waveform contenders for 5G - Suitability for short packet and low latency transmissions, in: Vehicular Technology Conference, VTC Spring, 2014 IEEE 79th, 2014, pp. 1-5. http://dx.doi.org/10.1109/ VTCSpring.2014.7023145.

[24] 3GPP TS 36.104. Base Station (BS) radio transmission and reception. 3rd Generation Partnership Project; Technical Specification Group Radio Access Network; Evolved Universal Terrestrial Radio Access (E-UTRA).

[25] V. Berg, J.B. Doré, D. Noguet, A flexible radio transmitter for TVWS based on FBMC, in: Digital System Design, DSD, 2013 Euromicro Conference on, 2013, pp. 163-167. http://dx.doi.org/10.1109/DSD.2013.25.

[26] V. Berg, J.B. Doré, D. Noguet, A flexible FS-FBMC receiver for dynamic access in the TVWS, in: 2014 9th International Conference on Cognitive Radio Oriented Wireless Networks and Communications, CROWNCOM, 2014, pp. 285-290. http://dx.doi.org/10.4108/icst.crowncom.2014.255866.

[27] 3GPP TSG RAN WG1, R1-163558, Way forward on assumptions for waveform evaluation, 2016.

[28] M. Matthe, L.L. Mendes, G. Fettweis, Asynchronous multi-user uplink transmission with generalized frequency division multiplexing, in: Communication Workshop, ICCW, 2015 IEEE International Conference on, 2015, pp. 2269-2275. http://dx.doi.org/10.1109/ICCW.2015.7247519.

[29] M. Fuhrwerk, J. Peissig, M. Schellmann, Channel adaptive pulse shaping for OQAM-OFDM systems, in: 2014 22nd European Signal Processing Conference, EUSIPCO, 2014, pp. 181-185.

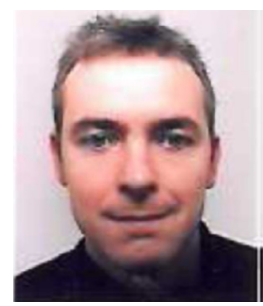

Jean-Baptiste Doré obtained his Masters degree in 2004 from the INSA and his Ph.D. in 2007. He started working at NXP semiconductors, home business unit, as a signal processing architect. He was in charge on the study and design of signal processing algorithms for DVB-T products. In 2009 he joined the Centre for Atomic Energy as a research engineer. His main research topics are signal processing, hardware architecture, PHY and MAC layers for wireless network.

Robin Gerzaguet received the degree in electrical engineering from GrenobleINP, France, in 2011 and the Ph.D. degree in both the Gipsa-laboratory (GrenobleAlpes University) and ST-Microelectronics in 2015. He is now with CEA-Leti where he works on physical layer and filter bank multicarrier waveforms in the context of millimeter wave transmissions. His research interests are in signal processing algorithms dedicated to communication, including channel estimation, synchronization, and digital RF impairments compensation. 
Nicolas Cassiau received the M.S. degree in Electronic Systems and Signal Processing in 2001 from Polytech'Nantes, Nantes, France. Since then he has been with CEA-Leti in Grenoble. His fields of interest are digital wireless communications and algorithms design. He has been working in particular on multiple antenna systems (MIMO), on the physical layer of OFDM systems and more recently on 5G, studying waveforms like FilterBank MultiCarrier and their application to millimeter wave frequencies. He has been involved in several European projects like 5GNOW, FANTASTIC5G and mmMAGIC.
Dimitri Ktenas received the Dipl.-Ing. degree in Electronics and Communications Engineering from ENSERG, Grenoble (France), in 2001. From that time, he has been with CEA-Leti where he is currently leading the Broadband Wireless Systems Lab. His main research interests are PHY, MAC and cross-layer optimization for 5G cellular networks. He has published $45+$ scientific papers in international journals and conference proceedings and 3 book chapters, and is the main inventor or coinventor of $10+$ patents. 